\title{
Safety of transcranial Direct Current Stimulation: Evidence Based Update 2016
}

\author{
Marom Bikson $^{1 *}$, Pnina Grossman ${ }^{1}$, Chris Thomas ${ }^{1}$, Adantchede Louis Zannou $^{1}$, Jimmy Jiang ${ }^{1}$, \\ Tatheer Adnan ${ }^{1}$, Antonios P Mourdoukoutas ${ }^{1}$, Greg Kronberg ${ }^{1}$, Dennis Truong ${ }^{1}$, Paulo Boggio ${ }^{2}$, \\ André R. Brunoni ${ }^{3}$, Leigh Charvet ${ }^{4}$, Felipe Fregni ${ }^{5}$, Brita Fritsch ${ }^{6}$, Bernadette Gillick ${ }^{7}$, Roy H. \\ Hamilton $^{8}$, Benjamin M. Hampstead ${ }^{9}$, Ryan Jankord ${ }^{10}$, Adam Kirton ${ }^{11}$, Helena Knotkova ${ }^{12}$, David \\ Liebetanz $^{13}$, Anli Liu ${ }^{14}$, Colleen Loo ${ }^{15}$, Michael A. Nitsche ${ }^{16}$, Janine Reis ${ }^{6}$, Jessica D. Richardson ${ }^{17}$, \\ Alexander Rotenberg ${ }^{18}$, Peter E. Turkeltaub ${ }^{19}$, Adam J. Woods ${ }^{20}$
}

${ }^{1}$ Department of Biomedical Engineering, The City College of New York, New York, NY

${ }^{2}$ Cognitive Neuroscience Laboratory and Developmental Disorders Program, Center for Health and Biological Sciences, Mackenzie Presbyterian University, Sao Paulo, Brazil

${ }^{3}$ Service of Interdisciplinary Neuromodulation, Department and Institute of Psychiatry, Laboratory of

Neurosciences (LIM-27), University of São Paulo, São Paulo, Brazil

${ }^{4}$ NYU MS Comprehensive Care Center, Department of Neurology, New York University School of Medicine, New York, NY

${ }_{5,17}$ Berenson-Allen Center for Noninvasive Brain Stimulation, Department of Neurology, Beth Israel Deaconess Medical Center, Harvard Medical School, Boston, Massachusetts, United States.

${ }^{6}$ Department of Neurology, University Medical Center, Freiburg, Germany

BrainLinks-BrainTools Cluster of Excellence, University of Freiburg, Germany

${ }^{7}$ Department of Physical Medicine and Rehabilitation, University of Minnesota Medical School, Minneapolis, MN

${ }^{8}$ Laboratory for Cognition and Neural Stimulation, University of Pennsylvania, Philadelphia, PA, USA

Center for Cognitive Neuroscience, University of Pennsylvania, Philadelphia, PA, USA

Department of Neurology, University of Pennsylvania, Philadelphia, PA, USA

${ }^{9}$ Mental Health Service, VA Ann Arbor Healthcare System, Ann Arbor, MI; Department of Psychiatry, University of Michigan, Ann Arbor, MI

${ }^{10}$ Applied Neuroscience, $711^{\text {th }}$ Human Performance Wing, Air Force Research Laboratory, WPAFB, OH

${ }^{11}$ Departments of Pediatrics and Clinical Neurosciences, Cumming School of Medicine, University of Calgary, Calgary, AB, Canada

${ }^{12}$ MJHS Institute for Innovation in Palliative Care, New York, NY; Department of Social and Family Medicine, Albert Einstein College of Medicine, The Bronx, NY

${ }^{13,16}$ Department of Clinical Neurophysiology, University Medical Center, Georg-August-University, Goettingen 37075, Germany

${ }^{14}$ NYU Comprehensive Epilepsy Center, New York University School of Medicine, New York, NY

${ }^{15}$ Professor, Psychiatry, University of New South Wales; Professorial Fellow, Black Dog Institute; Clinical Academic, St George Hospital; Sydney, Australia

${ }^{16}$ Leibniz Research Centre for Working Environment and Human Factors at the TU Dortmund, Dortmund, Germany

Department of Neurology, University Medical Hospital Bergmannsheil, Bochum, Germany

${ }^{17}$ Department of Communication Sciences \& Disorders, The University of South Carolina, Columbia, SC, USA; Department of Speech and Hearing Sciences, The University of New Mexico, Albuquerque, NM

${ }^{18}$ Berenson-Allen Center for Noninvasive Brain Stimulation, Division of Cognitive Neurology, Department of Neurology, Harvard Medical School and Beth Israel Deaconess Medical Center, Boston, MA, USA Pediatric Neuromodulation Program, Division of Epilepsy and Neurophysiology, Department of Neurology, Children's Hospital Boston, Harvard Medical School, Boston, MA, USA

${ }^{19}$ Department of Neurology, Georgetown University, Washington, DC

Research Division, MedStar National Rehabilitation Hospital, Washington, DC

${ }^{20}$ Center for Cognitive Aging and Memory, Institute on Aging, Department of Aging and Geriatric Research, McKnight Brain Institute, University of Florida, Gainesville, FL

*Corresponding author: bikson@ccny.cuny.edu, Department of Biomedical Engineering, The City College of New York of CUNY, T-403B, 160 Convent Avenue, New York, NY 10031 


\section{Abstract}

This review updates and consolidates evidence on the safety of transcranial Direct Current Stimulation (tDCS). Safety is here operationally defined by, and limited to, the absence of evidence for a Serious Adverse Effect, the criteria for which are rigorously defined. This review adopts an evidence-based approach, based on an aggregation of experience from human trials, taking care not to confuse speculation on potential hazards or lack of data to refute such speculation with evidence for risk. Safety data from animal tests for tissue damage are reviewed with systematic consideration of translation to humans. Arbitrary safety considerations are avoided. Computational models are used to relate dose to brain exposure in humans and animals. We review relevant dose-response curves and dose metrics (e.g. current, duration, current density, charge, charge density) for meaningful safety standards. Special consideration is given to theoretically vulnerable populations including children and the elderly, subjects with mood disorders, epilepsy, stroke, implants, and home users. Evidence from relevant animal models indicates that brain injury by Direct Current Stimulation (DCS) occurs at predicted brain current densities $(6.3-13 \mathrm{~A} / \mathrm{m} 2)$ that are over an order of magnitude above those produced by conventional tDCS. To date, the use of conventional tDCS protocols in human trials $(\leq 40$ $\min , \leq 4 \mathrm{~mA}, \leq 7.2$ Coulombs) has not produced any reports of a Serious Adverse Effect or irreversible injury across over 33,200 sessions and 1,000 subjects with repeated sessions. This includes a wide variety of subjects, including persons from potentially vulnerable populations. 


\section{Introduction}

Table of Contents

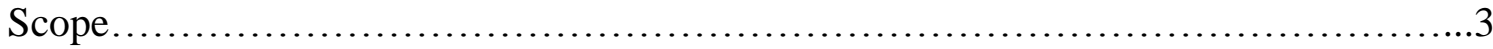

Definitions and Considerations of Dose Metrics for tDCS Safety.................................6

Definition and Considerations of Serious Adverse Effects for tDCS Safety.............12

Assumptions Regarding Dose-Response Curves for Safety Data from Animal Studies...17

DCS Safety Data from Animal Lesion Studies and Translational Models................18

tDCS Safety Data from Human Trials and Models...................................22

Safety Considerations for Specific Theoretically Susceptible Groups or Situations:

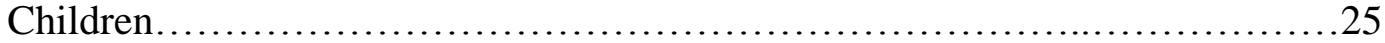

Aging Populations ..................................................29

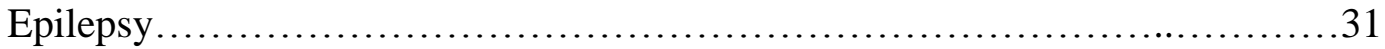

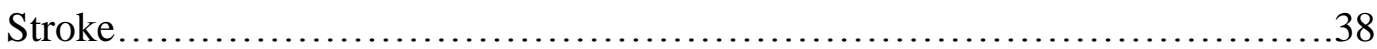

Mood Disorders.......................................................42

Subjects with Implants.................................................43

Home Use............................................................44

Conclusions.................................................................. 48

Scope

The goal of this report is to update the state-of-the-art on the safety of transcranial Direct Current Stimulation (tDCS), based on published Serious Adverse Effects in human trials 
and irreversible brain damage in animal models. For the purposes of this report, tDCS includes non-invasive transcranial electrical stimulation using direct current with a sustained intensity of a few $\mathrm{mA}$ and duration of up to tens of minutes; with specific definitions and inclusion/exclusion criterion defined. Basing our evaluation solely on established evidence, we rely on 1) testing in human trials, including reports of serious adverse events and imaging changes; 2) animal models, including histologically observable tissue; and 3) computational modeling to the limited extent it can inform the interpretation of experimental data. By consensus, we distinguish between adverse events (which are potentially coincidental) and adverse effects (which are believed to be causally related to stimulation), examining specific case data taken in the context of best experimental practices and all available tDCS data. Tolerability or transient adverse cognitive and behavioral changes that are not associated with Serious Adverse Effects are not taken into account.

Electrical stimulation in animals is referred to as Direct Current Stimulation (DCS; even when epicranial), as opposed to tDCS, to distinguish it from the human stimulation. For human data, the review has the limitation that it relies largely on reports of serious adverse events in published controlled studies in which subjects are not typically exhaustively tested for injury or followed for an extensive period. Prospective studies on tDCS safety are limited in humans [1][2]. For animal data, effort is devoted to understanding the translation of findings (e.g. dose scaling) to humans. Data from translational animal models are taken to support establishing tDCS safety limits only in the context of irreversible brain damage. We avoid speculation regarding theoretical risks 
of tDCS that are based on extrapolation from reports in which no specific link to tDCS has been established (e.g. inferring the potential risks of low intensity direct current based on the known risks of high intensity current).

Exclusion of subjects with preexisting co-morbidities from participation in clinical trials (e.g. exclusion of subject with depression from stroke studies, and exclusion of subjects with stroke from depression studies) reduces the number of complicated cases tested with tDCS. When such exclusion is not explicitly justified for safety reasons then it likely reflects experimental design (e.g. depression post stroke is considered a different illness to depression of another etiology) rather than concern regarding risk. Nonetheless, such "conservative" exclusions, as well as subject-specific safety monitoring protocols applied in the absence of evidence for risk, can be a source of confusion with regards to safety norms and are therefore discussed in this review.

Operator intentions when applying tDCS, the efficacy of tDCS in eliciting desired outcomes [3], and the presumed mechanisms of tDCS are not relevant for the scope of this review [4]. Similarly, potential neuroprotective effects are not within our scope [5], except for instances in which they inform safety. Animal safety data are limited to noninvasive or epicranial electrode techniques, since the safety profile for electrodes that directly contact with brain tissue is distinct (e.g. electrochemical in a way not relevant for non-invasive techniques). This review aggregates and analyzes data relevant to the safety of tDCS and comments on experience in human trials of tDCS to date. It does not make specific recommendations for protocols or serve as a guideline for the design of safety 
protocols. This review may, however, inform ongoing ethical and regulatory decisions [6].

Definitions and Considerations of Dose Metrics for tDCS Safety

For the purposes of this review, tDCS is defined as a technique in which the dose [7] is a waveform of single sustained direct current (DC), with the exception of one ramp-up and one ramp-down period, applied to the head using at least one cephalic electrode. tDCS is non-invasive and requires appropriate electrolyte buffer (conductive gel, paste, or saline) between the electrode and the skin. tDCS thus does not include the use of subdural stimulation electrodes.

While tDCS could technically include any waveform that does not change polarity (e.g. even a monophasic triangle wave), tDCS as used across current human trials involves only fixed sustained direct current. The lower-case " $\mathrm{t}$ " in tDCS is thus important to emphasize a proper name that designates a specific stimulation approach. Hence trains of monophasic pulses are not tDCS as defined here (but rather transcranial Pulsed Current Stimulation, even when a DC offset is included). Similarly neither oscillating transcranial direct current stimulation (a monophasic square waveform) nor a rectified or monophasic sinusoidal waveform is included in tDCS as defined here.

Any electrode from which current enters into the body is an anode, and any electrode where current exits the body is a cathode. tDCS/DCS must have at least one anode 
electrode and one cathode electrode. In the tDCS literature, "anodal-tDCS" and "cathodal-tDCS" is used to describe electrode placement in relation to the brain region primarily targeted along with the direction of the intended effect (e.g. "anodal motor cortical tDCS" is intended to increase motor cortical excitability) - but these expressions should be used with discretion. Under either polarity electrode, the direction of measured excitability changes can vary with brain state and dose parameters such as stimulation intensity and duration [8],[9],[10]. In addition, this common terminology might be misleading in the context of safety concerns, since both polarity electrodes are always present and since all current entering the cortex must exit, passing through intermediary brain regions [11]. Moreover, cortical folding (sulci and gyri) results in cortical current flow polarity inversions with respect to the cortical surface even under a single electrode [12]. Therefore, we do not attempt to develop separate safety criterion for "anodal" or "cathodal" tDCS. For the purposes of aggregating number of stimulation sessions, "anodal" and "cathodal" tDCS are collapsed, and tDCS/DCS safety data across polarities are generally grouped except where there are specific hypotheses to consider polarity specific effects. For animal DCS studies we collapse across polarities to obtain a conservative injury estimate. Differences in the two polarities in regard to injury thresholds and mechanisms are expected depending on the mechanism of injury, but here we consider the minimum threshold at any polarity. An exception to collapsing across polarities is in our discussion of testing on seizures, where for reasons explained below the positions of the cathode and anode are noted. 
tDCS/DCS intensity (in amperes, A or mA) is the steady-state intensity applied to the anode (opposite to cathode). If multiple electrodes are used, intensity is the sum of the current at all anodes (opposite of the sum at all cathodes). It is expected that the use of multiple electrodes will influence stimulation outcomes even for the same total current (similarly to changing montage with fixed current), but see cautions on data aggregation below. tDCS/DCS duration (in seconds or minutes) indicates the length of time current is at the steady level, and excludes the ramp-up and ramp-down periods, that usually last 10-30 seconds for studies using minutes of stimulation.

The dose of a single tDCS/DCS session is defined by the electrode montage (skin contact area/size and position of all electrodes), stimulation intensity and duration (see [7] for dose definition). All other metrics are derivative, in the sense that they are determined by dose, and in some cases by dose and tissue properties. tDCS/DCS is current controlled, meaning the voltage is varied to maintain a fixed current, typically under $20 \mathrm{~V}$ [13], though much of this voltage (especially any time-dependent component) may reflect the electrode and skin impedance. Current density, as used in the literature, indicates the average current density (in $\mathrm{A} / \mathrm{m}^{2}$ ) at the electrode calculated by taking the applied current to a given electrode and dividing by electrode area. Average current density is not necessarily indicative of peak current density at the electrode (which may be concentrated at edges or spots; [14]) or in the brain (which depends on many other factors namely head anatomy; [15]). Stimulation charge (in coulombs, C) is determined by multiplying current by duration. Stimulation charge density $\left(A^{*} t / \mathrm{m}^{2}=\mathrm{C} / \mathrm{m} 2\right)$ is charge divided by electrode area, and is also an average metric. Stimulation power (in watts. $\mathrm{W}=\mathrm{V}^{*} \mathrm{~A}$ ) is 
voltage multiplied by current. Stimulation energy (in joules, $J=V^{*} A^{*} t$ ) is power multiplied by duration. For any tDCS session, the above "summary" metrics are a single number, or a single number per electrode, and determined fully and only by dose, as defined above.

We review data from human trials of tDCS by dose (Figure 2). Meta-analyses of tDCS inevitably collapse across various testing conditions ([16]; e.g. $1 \mathrm{~mA}$ intensity in adults with epilepsy using $25 \mathrm{~cm}^{2}$ electrodes vs. $1 \mathrm{~mA}$ intensity in healthy children using $35 \mathrm{~cm}^{2}$ electrodes). It is conventional to assume that risk increases monotonically with current intensity or duration (e.g. all else being equal, decreasing current from $2 \mathrm{~mA}$ to $1 \mathrm{~mA}$ maintains or reduces theoretical risk). In some sections, we aggregate data by current and/or duration (e.g. total number of sessions at $1 \mathrm{~mA}$ and 10 minutes), and we assume a monotonic dose-response relationship (e.g. the safety of $1 \mathrm{~mA}$ is supported by the total number of sessions at $1 \mathrm{~mA}$ or more). However, this collapsing of data is inherently problematic precisely because it ignores all other factors such as pre-existing morbidity. This report addresses some of these risk factors by considering theoretically vulnerable (susceptible) populations. Nonetheless, we believe aggregating all tDCS trials provides a general sense for the extent of up-to-date experience in the field. We further believe that our data-driven approach provides a basis for more specific analysis, and for future development of safety guidance recommendations.

Prior efforts proposed safety standards based on summary metrics [17],[18] such as charge (which combines stimulation intensity and time), current density (which combines 
intensity and electrode size), or charge density (which combines current, duration, and electrode size). On the one hand, summary metrics are appealing because they simplify analysis; for example $1 \mathrm{~mA}$ with 10 minutes, $2 \mathrm{~mA}$ with 5 minutes, and $10 \mathrm{~mA}$ for 1 minute are equivalent from the perspective of charge. However, basing safety standards on summary metrics presupposes that critical details are not lost in combining terms such as current and duration - and moreover assumes no complex interaction between dose terms and other factors such as inclusion criteria. This assumption is further complicated by the absence of established mechanisms for injury, which makes it difficult to know which stimulation properties are most relevant to safety. Thus, while useful in other contexts, safety discussion based on summary metrics is limited in this review.

Additional "distributed" metrics of stimulation can be predicted based on the underlying tissue properties and are not single values, but rather distributed values specific to locations within the brain. These include current density (in $\mathrm{mA} / \mathrm{m} 2$ ) that reflects the current flow distribution and intensity through the body. For the purposes of this review we consider current density in the skin relative to the brain. Electric field (in V/m) is current density multiplied by local tissue resistivity. The peak current density or electric field represents the maximum value at any point in space, which can be further restricted by head region such as peak current density in the brain or skin. Power density (in $\mathrm{mW} / \mathrm{m} 3$ ) is electric field multiplied by current density. The electric field predicts neuronal activation threshold more meaningfully than current density, but it is very sensitive to assumptions on local tissue resistivity. It is not established whether injury is 
linked to neuronal activation (e.g. excitotoxic). The above tissue properties are not time dependent, but can be combined with time in new metrics.

For the purposes of this safety review, we restrict inclusion of human trials with tDCS to those with "conventional" protocols (e.g. waveform intensities and durations). Conventional current intensities span $0.1 \mathrm{~mA}$ (used occasionally as a sham) to $4.0 \mathrm{~mA}$, with the majority of studies applying $1.0 \mathrm{~mA}$ and $2.0 \mathrm{~mA}$. Conventional durations span from 4 seconds (used only for transient changes; [19] to 40 minutes (>10 minutes of stimulation are commonly used to elicit durable changes). Conventional charge (reflecting duration and intensity) is limited to $7.2 \mathrm{C}$ (e.g. 40 minutes, $3 \mathrm{~mA}$ ). Studies with atypical preparation techniques, that may or may not be hazardous, are excluded, for example those using water rather than saline saturated sponges (see below). The inclusion or exclusion of a study for our purposes is not an endorsement or critique of any dose or methodology, but reflects the inevitability of establishing some practical boundaries for our purposes. That being said, no study which self-identified as tDCS, and met all other inclusion criterion, used a dose above our range.

tDCS studies in which electrodes were not prepared following established methods are excluded. Stimulation is applied over skin that is not compromised by a pre-existing burn or injury (e.g. open wound) and is thus largely homogenous; but acne is typically not an exclusion for electrode locations. Skin preparation does not typically include significant abrasion (intended to remove epidermis; [20]), though cleaning of the skin/hair with saline or alcohol is sometimes used [21]. Standard tDCS electrodes (pads) are typically 
square $5 \times 5 \mathrm{~cm}$ or $5 \times 7 \mathrm{~cm}$, though both smaller and larger electrode assemblies have been explored [22], as well as circular pads. Standard tDCS electrode assemblies use either metal or conductive rubber electrodes [14]. Electrolytes are most commonly isotonic saline (saturated in a sponge that wraps around the electrode), but conductive gels and/or creams have also been used. The details of electrode assembly design are considered important for tolerability and skin safety. For example, it is important to maintain a minimal distance between the electrode and skin, as well as the area of the electrode compared to the electrolyte-skin area [14]. Pad electrodes, by virtue of size and materials, are typically limited in number to a maximum of 3-4. High-Definition (HD) electrodes are circular $<1 \mathrm{~cm}$ diameter with a sintered $\mathrm{Ag} / \mathrm{AgCl}$ electrode and conductive gel or paste [23]. A higher number and density of HD electrodes may be used [24],[25]. When one or more HD electrodes are used, tDCS is called High-Definition tDCS (HD-tDCS) regardless of the number of electrodes or if stimulation is optimized for focality or intensity [26]. Except when indicated, our analysis is not specific to electrode design (e.g. HD-tDCS is "conventional" as long as meeting current, duration, and charge limits).

\section{Definition and Considerations of Serious Adverse Effects for tDCS Safety}

In this review, tDCS safety indicates the absence of a Serious Adverse Effect including brain tissue injury related to tDCS application. It is necessary to precisely define this threshold for safety for clinical trials and separately for experiments in translational animal models. 
For clinical trials, based on International and US guidelines on serious adverse events from medical devices (including the Office of Human Research and Protection (OHRP) of the U.S. Department of Health And Human Services (HSS); FDA regulations at 21 CFR 312.32[a]; 1996 International Conference on Harmonization E-6 Guidelines for Good Clinical Practice; ISO/DIS 14155--Clinical investigations of medical devices in humans, good clinical practices, 2008), we classify a Serious Adverse Effect related to tDCS as a documented event that:

1) Based upon scientific judgment is determined to be caused or aggravated by the application of direct current to the head, such that serious adverse events not linked to stimulation are excluded, even if they are subject to reporting requirements AND

2) Results in irreversible damage of brain tissue $O R$

3) Results in persistent disability or incapacity that produces an unwanted and substantial disruption of a person's ability to conduct normal life functions, i.e., the adverse effect resulted in an unwanted significant, persistent or permanent change, impairment, damage or disruption in the patient's body function/structure, physical activities and/or quality of life OR

4) Results in unexpected inpatient hospitalization or prolongation of existing hospitalization, where emergency room visits that do not result in admission to the hospital should be evaluated for one of the other serious outcomes (e.g., lifethreatening; required intervention to prevent permanent impairment or damage; other serious medically important event) OR 
5) Results in death or is life-threatening where the patient was at substantial risk of dying as a result of the adverse event, or use was discontinued based on evidence tDCS might have resulted in death OR

6) Medical or surgical intervention was necessary to preclude permanent imminent impairment of a body function due to tDCS, or prevent permanent damage to a body structure due to tDCS

A report meeting any of criteria 2 through 6 , but not 1 , would be a serious adverse event. Absence of a reported serious adverse event indicates lack of evidence for a Serious Adverse Effect. For a Serious Adverse Effect a causal link with tDCS is required - for example serious adverse events potentially related only to a pre-existing condition or other activity in the trial (e.g. a fall unrelated to stimulation) would not meet the above criteria. Similarly, study dropouts are not necessarily Serious Adverse Effects. Reversible skin irritation not requiring medical intervention to prevent permanent injury would not meet the above criteria. Sensation and transient pain (tingling, itching) are similarly not relevant for safety though they impact tolerability. Changes in clinical symptoms are not considered a Serious Adverse Effect, unless proven to meet the above criteria; nor are transient decrements in cognition or behavior [27].

Theoretical long lasting changes in neuronal morphology (e.g. spine density, synaptic plasticity) or electrographic activity (e.g. alpha oscillation power, ERP magnitude) are not considered a Serious Adverse Effect unless proven to meet the above criteria. Clinical 
and animal studies exploring long lasting changes remain an important but challenging area of research.

Because establishing causality, and thus meeting criteria for a Serious Adverse Effect is difficult, human subjects protection protocols often adopt predetermined and measurable stopping criteria to manage adverse events. Specific rules for subject withdrawal or trial-cessation are designed to minimize risk in a real-time manner, which is distinct from a trial designed to establish safety. For example, in a trial of tDCS for epilepsy, stop criteria may include: 1) discontinuation of the session if the frequency of interictal discharges or seizures increases by $50 \%$ above baseline in the 1 hour after stimulation or 2) cessation of the study if over $50 \%$ of subjects in a stimulation study have a $50 \%$ increase of seizure frequency in the first 24 hours after tDCS. Such rules provide greater objectivity for an investigator deciding whether a given event was serious enough to potentially cause harm to the patient regardless of causality, and thus errs on the side of safety. Then, in later analysis and discussion, a determination of probable causality can be decided - however as defined here, subject withdrawal or session/trial stop for a serious event is not necessarily a Serious Adverse Effect until causality is established.

We note that in some cases insufficient data are collected, or details reported, to confirm causality of adverse events or whether a dropout might relate to a Serious Adverse Effect. One mid-study termination of participation illustrates the desirability of more detailed reporting of adverse events and dropouts to determine whether or not they meet Serious 
Adverse Effect criteria. In this case [28], one patient's participation was discontinued because the person "became emotional" and "felt morose". While authors state they could not establish a causal relation between the emotional state and tDCS, the details of that investigation are not reported, nor is follow up to confirm that the patient recovered from this state. Although it seems unlikely that this patient experienced "an unwanted significant, persistent or permanent change, impairment, damage or disruption in ... quality of life," more details would help confirm whether this reported change was 1) severe and long-lasting enough to be considered a serious event, 2) indeed a change and not a previous/existing condition, and 3) caused or aggravated by application of tDCS. As such, this case does not meet our reports standard for a Serious Adverse Effect.

If tDCS is applied with the intention to produce an abnormal brain state, for example to interrupt normal brain processing, then the abnormal brain state would be expected and appropriate safety measures would be in place if needed (e.g. hospitalization), and this is not a Serious Adverse Effect. In this sense (extrapolating from related fields and standards) the intentional generation of a seizure by electroconvulsive therapy (ECT) or magnetic seizure therapy (MST) is not a Serious Adverse Effect, while the unintentional generation of seizures in rTMS is a Serious Adverse Effect.

We acknowledge that one potential limitation of using evidence-based causality to establish the criterion of Severe Adverse Effect is that it may not be possible to empirically determine whether causal relationships exist between very rare events and tDCS in the absence of sufficient amount of data. In the absence of sufficient data to 
establish these links, the causality criterion of severe adverse effects may theoretically obscure very uncommon but causally related events, creating a bias toward judging tDCS to be safe. None-the-less, sham-controlled trials are the best way to empirically assess adverse effects, including serious ones; and this review addresses the scale of data collected to date.

Assumptions Regarding Dose-Response Curves for Safety Data from Animal Studies

This review avoids specific recommendations or endorsements, but rather focuses on aggregating data and presenting the state-of-the-art in understanding on safety. This section discusses several dose response curves with a specific focus on aggregated animal data (next section). Different possible dose response curves for safety are illustrated in Figure 5 .

In summarizing animal safety data, the approach adopted here was to use the lowest current intensity documented to produce a measurable destructive brain tissue response in an animal model (illustrations: Figure 5A) at any stimulation duration. This approach has its own limitations and assumptions. In any given experimental series, the limitations on both the precision of current increments tested and the number of animals tested will limit validation of a single lowest damage threshold. Alternatively, the entire data set may consolidate a curve-fit to extrapolate a minimum damage threshold. Though the quality of curve fit may support this approach, assumptions on the type of dose-response curve for damage (Figure 5B) will profoundly influence the resulting extrapolation, notably to 
low doses not actually tested. For example, dose-response projections based on injury at moderate intensity would ignore if lower intensities might in fact provide protection from injury (i.e. so called hormetic dose response; [182], [183]). Especially in the absence of a mechanistic explanation for damage supporting a particular dose-response curve, and accumulation of data from different model systems and varying lesion measures we avoided extrapolation beyond tested stimulation intensities. For the same reason, we avoided putative summary-metrics of damage, such as charge or charge-density; but as the animal trials cited used stimulation durations equal to or greater than clinical tDCS, limits based simply on current can be considered conservative in regards to summary metrics influenced by time - assuming a monotonic relationship between stimulation duration and injury at any given intensity.

Additional assumptions about the dose-response relationship are made. Experimental studies are often limited in time points for measurement (since the collection of tissue for analysis often requires terminal procedures) so we assume that damage is irreversible and also delayed damage responses cannot be excluded. Again without an established mechanism for damage, we limit ourselves here to reported data.

The sensitivity of damage detection is evidently limited by the experimental measures. In addition, the relative sensitivity of animal tissue to DCS versus human tissue to tDCS injury is unclear. While arbitrary safety factors are sometimes applied in developing guidance, our goal here is to summarize injury evidence. In developing human safety guidelines it is prudent not to approach injury thresholds, especially given montage and 
inter-individual differences (Figure 1). Consolidated animal DCS safety data, and scaling to the human case using computational models, indicates that at least in regards to manifest tissue damage, current conventional tDCS protocols are orders of magnitude below threshold.

\section{DCS Safety Data from Animal Lesion Studies and Translational Models}

Data on DCS lesion threshold in animals have been used to support the safety of existing tDCS protocols; evidence demonstrating the wide gap between current tDCS protocols and DCS lesion thresholds provides some reassurance [17], [38]. However, with increasing adoption of tDCS, these data warrant updating.

The issues when basing human safety standards on animal histology thresholds were previous outlined [1] and include: 1) potential differences in susceptibility of animal and human tissue to damage; 2) experimental limits on detecting various modes of damage including assumptions about dose-response relationships; 3) difference in scale from rodent (or other nonhuman) to human gross anatomy; 4) difference in method of stimulation (e.g. transdermal vs. epicranial). The use of animal models provides the distinct advantage of being able to histologically assess the impact of current on brain tissue. The results from animal models can thereby inform current threshold limits and, in addition, be used to validate and improve the computational models used for determining predictive safety thresholds in humans. 
Tissue damage from animal studies using electrodes in direct contact with the brain or using varied waveforms (e.g. AC) are inappropriate (and potentially misleading) in regards to tDCS safety [38]. Here, results from three groups of testing safety thresholds for epicranial DCS are consolidated, acknowledging the limitations of the different methods of lesion detection (e.g. H\&E staining is potentially less sensitive than the direct staining of neurodegeneration by Fluoro-Jade C): 1) Liebetanz and colleagues [17]; 2) Fritsch and colleagues (unpublished data); 3) Jankord and colleagues (unpublished data). In all cases DCS was applied to the surface of the rat skull using a relatively small electrode-contact (defined as the electrolyte-skull interface) compared to the return electrode on the body. The lowest DCS intensity at which histological damage was recorded in the three studies were as follows: 1) Liebetanz. $500 \mu \mathrm{A}$ applied through 2.1 $\mathrm{mm}$ diameter circular electrode-contact for 10 minutes (return electrode on the chest), assessed by hematoxylin-eosin (H\&E) stain (used for histological assessment of tissue following current exposure); 2) Fritsch, $600 \mu \mathrm{A}$ applied through $4 \mathrm{~mm}$ diameter circular electrode-contact for 20 minutes (return electrode on the chest), assessed by FluoroJade C stain; 3) Jankord $500 \mu \mathrm{A}$ applied through $5 \times 5 \mathrm{~mm}$ square electrode-contact for 60 minutes (return electrode behind the neck), assessed by H\&E stain.

To scale these results to humans, we developed a high-resolution rat model and predicted brain current flow produced for each montage used (Figure 3). The predicted minimum induced current density for detected damage was 12,17 , and $6.3 \mathrm{~A} / \mathrm{m} 2$ for Liebetanz et al., Fritsch et al., and Jankord et al., respectively. By comparing resulting peak current density (or electric field) per applied $\mathrm{mA}$ in the rat brain to the peak electric field 
produced per $\mathrm{mA}$ in the human brain, we are able to propose a scaling factor. Specifically, the scaling factor allows us to predict how much current should be applied in the human using a representative montage (M1-SO adult) to approximate the brain electric field produced in a rat for a given current. Note the M1-SO montage is among the most commonly used in tDCS but does not produce the theoretically maximum brain electric field. Applying this scaling factor to the current damage threshold observed in rat allows us to predict a current intensity damage threshold in humans. The scaling factor determined was 288 for Fritsch and colleagues, 240 for Liebetanz and colleagues and 134 for the Jankord and colleagues studies. Combining the reported currentthresholds for damage in animal models with the respective rat-to-human scaling factors results in a predicted human damage threshold of $173 \mathrm{~mA}$ based on Fritsch, $120 \mathrm{~mA}$ based on Liebetanz, and $67 \mathrm{~mA}$ based on Jankord. These scaled values are over an order of magnitude above maximum currents levels used during tDCS. Differences across animal models are expected and arise from additional dose metrics (e.g. time, which model based scaling does not account for), which are neglected for the purpose of the review. If separate scaling factors are used (e.g. average electrode current density as opposed to model based scaling) or additional dose metrics considered, then different animal-to-human scaling factors would be predicted for each study. This analysis does not in any way constitute an endorsement for the use of such high current in humans but serves only to illustrate the range between observable tissue damage in animal models and conventional tDCS. 
Kim et al. [5] assessed whether anodal DCS increases pre-existing infarct volume in a rat stroke model 2 days post-injury. Their results showed no increase in volume at the doses tested $\left(0.785 \mathrm{~cm}^{2}\right.$ epicranial electrode, $100 \mathrm{uA}$ for 20 minutes $)$, and a potential neuroprotective effect. Cathodal DC at $200 \mathrm{uA}$ has also been shown to have a protective effect for ischemic stroke in rats [184]. These results suggest that the safety threshold predicted above extends to post-injury models. Results in the mouse model differ from those in the rat. Peruzzotti-Jametti and colleagues [185] suggest anodal stimulation induced an increase in the post-ischemic lesion volume and augmented blood brain barrier derangement in a mouse model with $1.2 \mathrm{~mm}$ diameter epicranial stimulation at $250 \mathrm{uA}$ for 40 minutes total, while cathodal stimulation had a protective effect. Importantly, decreased mouse head volume compared to the rat suggests a further scaling factor which if $\geq 2$ brings this result in line with those in healthy rats. However, it is important to note that several studies in the acute and subacute phase of recovery have been successfully conducted in humans (see above) without reported serious adverse events [186], [152], [153], [187], [188].

\section{tDCS Safety Data from Human Trials and Models}

There is direct support for the safety of tDCS as applied thus far in controlled human trials (previously reviewed in [3],[29]). Mild skin erythema is common during tDCS and is not inherently hazardous [30] and resolves after stimulation. tDCS was not found to produce edema or injurious alterations of the blood-brain barrier or cerebral tissue detectable by MRI [2], though non-injurious reversible changes in brain perfusion are 
plausible [31],[32] as a result of direct action on endothelial cells or indirectly via modulation of neuronal (metabolic) activity.

During tDCS the ratio of current density in the skin to the brain is predicted to exceed 10:1 (Figure 1; [33]). If one assumes comparable sensitivity to injury of skin and brain, then the tolerability to tDCS evidenced by lack of skin lesions provides indirect support for safety with respect to the brain. For example, tDCS produces negligible temperature changes in the skin [23], making direct injury from brain heating improbable. Poor electrode skin contact (dry sponges) will lead to skin irritation (e.g. by dramatically reducing the stimulation area). In rare cases, poorly designed or prepared electrodes produced skin lesions [34],[35],[36],[37]; if these are attributed to electrochemical reactions produced locally at the electrode [38], [23], it would not be relevant for brain injury since chemical products above the skin surface are not expected to diffuse to the brain. Important factors in electrode design and preparation have been reviewed [39].

For a given applied current, the maximum current density generated in the brain will vary according to montage and head anatomy; the resulting current density can be quantified using computational models (Figure 1) and demonstrated experimentally [40]. In susceptible populations current flow to the head may be further altered by pathological changes in the cranium or brain tissue such as stroke, as may the case in patients with post-surgical or trauma induced skull defects or post-stroke encephalomalacia [41], [42]. Further divergence from expected current flow may be attributable to an immature brain anatomy in children [43], [44]. Across heads and montages (Figure 1) the maximum 
predicted brain current density $\left(0.23 \mathrm{~A} / \mathrm{m}^{2}\right.$ for a small adult head and $0.32 \mathrm{~A} / \mathrm{m}^{2}$ for pediatric head) remains substantially below injury threshold levels in animals (6.3-17 $\mathrm{A} / \mathrm{m}^{2}$, described in detail below). Peak current densities in the modeling literature (spanning heads, model parameterization and tDCS dose) range from 0.0828 to 0.211 $\mathrm{A} / \mathrm{m}^{2}$ [45], [46], [47], [48]. At these predicted current densities, we are not aware of any well-defined theoretical risk for brain injury by tDCS based on experiments or modeling.

Controlled human studies involving the general population, susceptible subjects (e.g. children), and potentially susceptible (e.g. subject with altered neuroanatomy or neurophysiology) populations support safety [49], [50], [51], [52]. We are aware of no direct evidence from human trials involving tDCS that suggests tissue damage or behavioral changes suggestive of irreversible brain injury. Though methodology and rigor for reporting adverse events in tDCS are inconsistent across studies [53], [54], [55], [29], [56], [57], [58], [59], [60] human trials (per IRB guidance) should have specifically designed safety monitoring and reporting. Especially given the severity of a serious adverse event as defined above and mandatory reporting requirements defined in CFR, the lack of Serious Adverse Effect report in any trial supports the absence of occurrence. A meta-analysis of the aggregate number of tDCS sessions (Figure 2) failed to identify even a single record of Serious Adverse Effect related to tDCS across $>33,200$ sessions. Among these over 1,000 subjects received tDCS repeatedly (multiple sessions across days) without Serious Adverse Effect. 
The acceleration in the number of publications is not associated with a general increase in trial size (number of subjects) or duration (number of sessions per trial) (Figure 6). This is consistent with tDCS being adopted by increasingly more independent groups as well increased activity within groups, and so more investigators in general. Demographics suggest a majority of sessions were applied to healthy subjects. This is consistent with the use of tDCS to study normal brain function under the assumption tDCS is minimal risk. The treatment of a broad range of indications has been explored by tDCS. Distribution of sessions by medical indications often reflects the size of trials rather than number of publications (e.g. tinnitus) (Figure 7). The distribution and diversity of clinical trials with tDCS support the generalization of overall safety findings.

There are also data on individual patients who have received over 100 treatment sessions of tDCS without any indication of adverse effects arising from cumulative exposure. These include a patient with schizophrenia who received maintenance tDCS once to twice daily on a domiciliary basis over a 3-year period (i.e.> 1000 sessions) [61]; and patients with depression who received multiple courses of tDCS (>100 sessions in total) safely, assessed with structured questionnaires of side effects and formal neuropsychological testing [59]. Further, thirty-three healthy volunteers received up to 30 sessions (6 weeks) of tDCS ( $2 \mathrm{~mA}, 20$ minutes, high-performance adhesive electrodes) without a serious adverse event [62].

To our knowledge, the US FDA considers trials of tDCS as non-significant-risk, which means tDCS is without reasonable expectation of any Serious Adverse Effect (as defined 
here). The FDA requires reporting of "unanticipated" adverse events. As of this date, the FDA "MedWatch" database search returns no reports for "tDCS" or "transcranial Direct Current Stimulation.” A similar research status approval is in place from Health Canada and internationally [6].

\section{tDCS Special Consideration for Safety in Children}

As is typical for most investigational techniques, experience with tDCS in children has been limited compared to adults and applications in the developing brain require additional considerations. Fewer than 5\% of published tDCS studies include pediatric populations. In children, considerations include potential modification of dosing for both safety and efficacy. Specific systems and techniques for recording, side-effects, potential adverse events and effects, and tolerability measures are required.

In trials involving children, at least 2800 sessions have been applied across nearly 500 subjects. No serious adverse effects have been reported. tDCS has been investigated in children with a variety of diagnoses including cerebral palsy, stroke, encephalitis, epilepsy, schizophrenia and attention-deficit hyperactivity disorder [76], [77], [78], [79], [49], [61], [80], [51], [50], [81], [82], [83]. According to clinicaltrials.gov, current studies in pediatric applications of tDCS include perinatal stroke, cerebral palsy, dystonia, childhood-onset schizophrenia, attention deficit hyperactivity disorder, and autism. The relatively limited nature of this tDCS experience across pediatric populations compared to adults is shown in Figure 7. 
Current flow modeling predict increased brain current density on average in children for the same applied dose (Figure 1), reflecting smaller average head sizes and possibly additional factors. However, conventional adult dosing still remains well below potentially hazardous levels defined by animal safety trials (see below). While reduced ( 1 mA) current intensities are often used in children, trials up to $2 \mathrm{~mA}$ [49] without serious adverse effects are reported. A tDCS modeling study performed in two typicallydeveloping children ages 8 and 12 years suggested higher peak electrical fields compared to adults at given current intensities with montage specific scaling [44]. In a pediatric tDCS modeling study, peak brain current flow and distribution were incorporated into modeling of brain electric fields of a child with perinatal stroke [43]. It was found that tDCS at $0.7 \mathrm{~mA}$ produced a peak brain current intensity comparable to an adult receiving 1.0 mA. This dose was subsequently applied in a bihemispheric montage in a pilot pediatric tDCS safety study in congenital hemiparesis [79]. Clinical neurophysiology in children indicated altered dose response, compared to adults, consistent with the scaling of fractional brain electric fields predicted by current flow models [84].

Specific to children with cerebral palsy, in 8 tDCS studies published between 2013 and June 2015, not one of the combined 176 children incurred a serious adverse event [79], [80], [50], [81], [82], [83], [85], [86]. Current intensities ranged from 0.7 to $2.0 \mathrm{~mA}$, with 9-20 minute sessions varying between single or serial sessions (10 maximum consecutive daily sessions). The most commonly investigated montage was M1-SO (7 of 8 studies), with one electrode over C3 (M1) and one electrode supra-orbital (SO), with either the 
anode or cathode over M1. The most commonly reported minor adverse events included the sensation of tingling or discomfort under the electrode sites, reported in both active and sham conditions. For example, in a single-session sham-controlled bihemispheric tDCS study involving 13 children aged 7-18 with congenital hemiparesis due to perinatal stroke, 1 child from the sham group reported a burning sensation, while another from the active group reported itching [79]. One child withdrew from the study due to discomfort during the 30-second ramp-up. In a study of serial sessions of tDCS in 13 children with dystonia, tingling was reported at the ramp-up phase of both real and sham stimulation [86]. Two children reported discomfort; a reduction of tDCS amplitude from 2.0 to 1.5 $\mathrm{mA}$ extinguished this sensation. In another tDCS study in 11 children with dystonia, one subject withdrew due to stimulation-related discomfort while another required adjustment of the current from $1.0 \mathrm{~mA}$ to $0.65 \mathrm{~mA}$ in order to reduce discomfort [80].

Pediatric-based studies are also examining the synergistic application of tDCS during rehabilitation sessions to enhance motor plasticity (clinicaltrials.gov \#NCT02170285). One study in children with congenital hemiparesis combines both constraint-induced movement therapy and tDCS. This serial-session trial applies tDCS in an M1-SO cathodal contralesional montage at an intensity of $0.7 \mathrm{~mA}$ for the first 20 minutes during a 2-hour rehabilitation session involving the more-affected hand (clinicaltrials.gov \#NCT02250092).

A randomized, controlled, clinical trial of 24 children aged 6-18 years with perinatal stroke and hemiparesis combined intensive motor learning therapy with tDCS. Subjects 
received contralesional M1 cathodal $1 \mathrm{~mA}$ tDCS (or sham) for the first 20 minutes of a two-hour therapy session for 10 consecutive weekdays. Examination of safety outcomes after 12 and 24 subjects, including function of both the paretic and unaffected upper limb, found no serious adverse effects. Aside from scalp tingling/itching (42\%), no other adverse events were reported (Kirton, unpublished). Additional case reports with no serious adverse events include a 16-year-old with childhood stroke and hemiparesis who received contralateral cathodal $1 \mathrm{~mA}$ stimulation for 10 days with therapy and a 15 -yearold with schizophrenia and refractory auditory hallucinations who received 2 weeks of superior temporal cathodal stimulation for 20 min daily with no adverse events (Kirton, unpublished).

A study of tDCS enhancement of motor learning in 24 typically developing children aged 6-18 years found no serious adverse effects (Kirton, under review). Children performed a motor learning task repeatedly over 3 days, randomized to sham, $1 \mathrm{~mA}$ contralateral M1 anodal, 1 or $2 \mathrm{~mA}$ ipsilateral cathodal M1 stimulation for the first 20 minutes of each training session. Specific safety outcomes included any decrease in either the trained or untrained hand as well as decline in multiple non-trained motor tasks before and after intervention. All functional outcomes improved with tDCS. Mild tingling or itching of the scalp was reported in $55 \%$ of subjects but never precluded participation.

Combining this emerging pediatric evidence with the larger animal and adult experience suggests tDCS within the same ranges of dosing and duration can be considered minimal risk, based on current evidence, in school-aged children. 
tDCS Special Considerations for Safety in Aging Populations

Given the increasingly older demographic of our national population, there is a growing interest in tDCS as a mechanism for stabilizing or even enhancing cognitive functioning in older adults [87]. It is established that particular aspects of cognitive decline, even during "normal" aging [88], are exacerbated by incipient neurodegenerative diseases. It is important to distinguish between increased risks arising from unrelated comorbidities in these subjects, such as increased risk of seizures and other comorbid medical conditions, including neurodegenerative disease [89], from evidence that aging subjects are at increased risk for Serious Adverse Effects during tDCS. As in other situations, exclusion of subjects from tDCS human trials for pre-existing conditions is not necessarily evidence in itself for increased risk.

Building on the results of a recent review in this content area [90], we identified a total of 19 tDCS studies that targeted a range of motor and cognitive abilities [91], [92], [93], [94], [95], [96], [97], [98], [17], [99], [100], [101], [102], [103], [104], [105], [106], [107],[108]. These 19 studies included over 500 participants whose mean ages were in the mid to late 60 's. Across studies, participants received between 1 and 10 sessions of tDCS, with a duration of 5 to 30 minutes, at an intensity of 1 to $2 \mathrm{~mA}$. Five studies failed to report any safety data [108], [91], [94], [104], [105] (which this review considers evidence for the absence of a serious adverse event), and one reportedly asked about side effects but failed to report any data [96]. None of the remaining 13 studies reported any 
adverse events (serious or otherwise). Five studies made limited comments about all participants tolerating treatment well [107], [102], [103], [106], [109], and the other eight reported expected sensory experiences (e.g., itching, tingling, burning) that were generally indistinguishable from those reported by participants receiving sham stimulation.

If effective, tDCS could be particularly beneficial for treating cognitive, motor, and psychiatric symptoms of neurodegenerative diseases, as well as decline associated with normal aging (see reviews by [90], [110]). We identified 15 studies that evaluated the effects of tDCS on patients with Alzheimer's disease [111], [112], [113], [114], [115], [116], Parkinson's Disease [109], [117], [118], [119], [120], [121], Dementia with Lewy Bodies [122], Corticobasal degeneration [123], and Frontotemporal dementia [124]. In all, there were over 275 patients (some assigned to sham conditions) who received between 7 and 30 minutes of stimulation in each of 1 to 10 sessions with an intensity of between 1 and $2.8 \mathrm{~mA} .10$ studies comment on safety. One patient was removed from treatment after experiencing delirium caused by pneumonia and another patient experienced a bout of diarrhea and could not attend some of the tDCS sessions [116]. Neither of these events appear to be attributable to tDCS and thus are not Serious Adverse Effects. Four studies reported typical side effects (i.e., itching, tingling, burning) [122], [116], [119], as well as temporary headache and dizziness [111]. It is also worthwhile to note that a review of eight tDCS studies in the geriatric depression literature found no major side effects of stimulation [125]. 
Overall, there were no unexpected or severe adverse events in over 40 studies with more than 600 older adults regardless of cognitive or disease status. Thus, there is no current evidence for increased risk of Serious Adverse Effects with aging subjects.

Risk of tDCS-related Seizures in Healthy Populations and Special Considerations for Safety in Epilepsy

We distinguish between epilepsy and seizure induction. As defined for our purposes, a Serious Adverse Effect for tDCS would include the triggering of a seizure, either in healthy individuals or individuals with epilepsy or others predisposed to seizures, with evidence that tDCS was causally related to the ictal event. Encouragingly, no such instances have been reported.

A more ambiguous topic in epilepsy neuromodulation in whether aggravation of epileptiform electrographic activity itself is a Serious Adverse Effect. One can imagine exacerbation of interictal epileptic spike frequency on EEG without change in the clinical picture and no new requirement for medical intervention. Per our definition this would not be a Serious Adverse Effect if there were no clinical (treatment) impact. Moreover, one needs to be conservative in extrapolating between a biomarker (interictal activity) and relevant clinical morbidity (seizures) ([126], [127]).

tDCS safety with respect to seizure induction is underscored by in vitro DCS studies indicating that the electric field threshold for modulating ongoing epileptiform activity 
$(\sim 1 \mathrm{~V} / \mathrm{m}$, consistent with a heightened sensitivity to excitability; [128]) is more than an order of magnitude lower than intensity thresholds for generation of activity in a quiescent brain slice $(>80 \mathrm{~V} / \mathrm{m}$; [129] which would require $>160 \mathrm{~mA}$ tDCS). An important question is whether the electric field threshold to generate epileptic activity in active (not quiescent) but not already epileptic tissue. To this end, it was further tested in vitro if ongoing brain gamma oscillations will reduce thresholds for electrographic seizure generation. In this experiment, the DCS intensity need to initiate epileptic activity (80-120 V/m) in an active (but non-epileptic) neuronal network, was also more than an order of magnitude above that generated in conventional tDCS $(<1 \mathrm{~V} / \mathrm{m})$ (Figure 4; Bikson et al., in preparation).

Across numerous in vitro seizure models, the polarity of stimulation producing somatic depolarization or hyperpolarization aggravates or inhibits activity [130],[131],[132], respectively. In vivo hyperpolarization inhibits activity while depolarization has no or mixed effects (see below). During tDCS placement, including for epilepsy of an anode/cathode over a brain region is typically intended to excite/inhibit. However, as discussed above, this concept is limited by 1) the ubiquitous presence of both electrodes, such that 'anodal tDCS' refers only to the nominal brain target being near the anode (a fact not negated by an extracephalic electrode, since current must pass in and out the brain) and 2) current flow under and between electrodes producing a locally alternating pattern of polarization [133]. Therefore, for the majority of this review we collapsed socalled "anodal" and "cathodal" tDCS in our analysis. We deviate from this convention in this section on seizures because of the strong polarity dependence show in animal 
models, which has been adopted in designing clinical trials - but none-the-less note presence and location of both electrodes.

Several in vivo studies have evaluated the safety and efficacy of stimulation in rodent epilepsy models and in human subjects with intractable focal seizures [76] where the functional polarity (anodal or cathodal) is assumed to be the epicranial electrode over the seizure focus and the other electrode is positioned on the torso or over a non-epileptic cortical region. In rats, Liebetanz found an intriguing capacity for seizure prophylaxis by tDCS where cathodal epicranial electrode stimulation increased threshold for focal seizures up to 2 hours. The same experiment indicated that anodal epicranial electrode stimulation had no effect on seizure threshold [9]. The antiepileptic potential of cathodal tDCS was also demonstrated in a rat amygdala-kindling temporal lobe epilepsy model where Kamida and colleagues demonstrated that cathodal tDCS reduced seizure severity and EEG after-discharge duration, while elevating the after-discharge threshold. This treatment regimen also corresponded to improved cognitive performance on the Morris water maze [134]. The Kamida group also identified an anti-convulsive cathodal tDCS effect in a rat pup lithium-pilocarpine status epilepticus model where tDCS appeared to mitigate status epilepticus-related cell hippocampal cell death and preserve performance on the Morros water maze spatial memory task [135]. In a different approach where tDCS was deployed after seizure onset, Dhamne and colleagues identified a potent antiepileptic effect of cathodal tDCS in a rat pentylenetetrzole (PTZ) status epileptic model, and also demonstrated that tDCS and lorazepam, a first-line anticonvulsant may act synergistically [130], [136]. Notably, while tDCS in this experiment was delivered in 
setting of ongoing seizures, neither seizure exacerbation nor increase in epileptiform EEG discharges were identified. This favorable result in a primary generalized epilepsy model is consistent with a prior report that epicranial cathode electrode tDCS transiently suppress spike and slow wave discharges on a rodent model of absence epilepsy [129].

In humans, clinical experience with tDCS in epilepsy indicates the procedure is well tolerated and safe [3]. When a cathode electrode is directed over epileptogenic cortex, there may be a mild anti-epileptogenic effect, manifest as reduced interictal discharge frequency [137]. There have been several studies investigating the effect of tDCS with the cathode positioned over the targeted cortex on reducing seizure frequency and focal hyperexcitability in partial onset seizures (six original studies published in English in a recently published meta-analysis, [137]). In a randomized, sham-controlled study applying a single session of tDCS ( $1 \mathrm{~mA}, 20 \mathrm{~min})$ with the cathode over the epileptogenic zone and the anode placed over an area without epileptiform activity, there was a significant reduction in the frequency of interictal epileptiform discharges, with a trend towards decrease in seizure frequency [138]. Likewise, in another study involving 36 children with partial epilepsy, a single session of tDCS (1 mA, $20 \mathrm{~min})$ with the cathode directed toward the target suppressed epileptiform activity for 48 hours and demonstrated a trend toward seizure reduction [78]. Several case reports and small case series similarly demonstrate safe and well-tolerated application of tDCS with the cathode over epileptogenic cortex [139], [140], including transient reduction in spike frequency in continuous spike and waves during slow wave sleep [141]. 
In addition to seizure suppression, tDCS may have a role in mitigating behavioral symptoms that are commonly comorbid with epilepsy. In a randomized controlled study of 37 patients the an anode was positioned over the left dorsolateral prefrontal cortex to reduce depressive symptoms, and the cathode over the right supraorbital area, to test the capacity of tDCS to treat comorbid mood symptoms in patients with temporal lobe epilepsy [142]. While stimulation was performed over a location distant from epileptogenic cortex, none of the subjects in the active stimulation group experienced an increase in seizure activity over a 4-week follow up, and interictal epileptic EEG activity remained unchanged.

As an example of the complexity in determining the causality of tDCS and seizures, especially in subject with epilepsy, we note a case report of a focal onset seizure during a tDCS trial [143]. A 4-year-old boy with a history of prematurity, left dominant spastic paresis, and infantile spasms, but seizure free for 2 years on anti-epileptic medication received serial stimulation to attempt to relieve left-sided spasticity (with one of the medications withdrawn 2 weeks prior to stimulation). The anode electrode (1.2 mA, 20 min) was directed over the right paracentral region, paired with escitalopram (to facilitate the serotonergically-mediated excitatory effect of tDCS). The cathode electrode location was not specified. Four hours after the third session of stimulation, the patient developed a partial onset seizure characterized by speech arrest, confusion, leftward eye gaze deviation, left arm clonic movements, and secondary generalization which required administration of intravenous midazolam. The patient's lateralized semiology suggested that the seizure onset was from the frontocentral region, corresponding to the region 
under the anode electrode. The application of an anode electrode, as opposed to a cathode electrode, over potentially epileptogenic cortex (in a patient with a history of probable generalized cortical hyperexcitability with superimposed focal hyperexcitability of the left paracentral region) is atypical. Moreover, regardless whether a cephalic or extracephalic cathode electrode position was used, there was no consideration of altered current delivery [44] and potentially inverted dose response [144] expected in children (see tDCS Special Consideration for Safety in Children). There was no consideration of further interactions between drugs and tDCS dose response [74]. It is unclear whether the interictal EEG performed was of adequate duration and proximity to the date of stimulation to provide appropriate reassurance that the patient did not have any ongoing interictal epileptiform activity, especially in the region of stimulation. In totality, regardless of insufficient attention to conventional safety considerations, this report does not meet our standards for a Serious Adverse Effect; our definition includes a requirement to establish reasonable causality, which is hampered in this case by insufficient monitoring and controls (e.g. medication withdrawal).

In repetitive Transcranial Magnetic Stimulation (rTMS), seizure provocation remains the primary safety concern in healthy individuals, and a diagnosis of epilepsy is typically considered an exclusion criterion for high-frequency repetitive TMS studies [145] but see [146]. Concerns about TMS have spread to tDCS research, such that it has become a common exclusion criterion for tDCS [3], simply by historical association with TMS and for experimental reasons (e.g. to minimize risks of coincidental seizures during a trial). However, in contrast to TMS, electric fields produced by tDCS in the brain are static not 
pulsed, and are two orders of magnitude below those employed in rTMS [12], [147]. Similarly, tDCS produces an electric field in the brain over two orders of magnitude below ECT and TES where hundreds of mA (e.g. $800 \mathrm{~mA}$ ) and pulse waveforms are needed to trigger an overt motor response [40], [148], [149]. Combined with preclinical studies on DCS seizure threshold and experience from hundreds of clinical trials, these data suggest no evidence or mechanistic support for seizure generation with conventional tDCS protocols.

Intense electrical stimulation can produce epilepsy in animal models through "kindling", where sessions of stimulation are sufficient to trigger seizures As noted above, the intensity of tDCS is far below the seizure induction threshold, and even much higherintensity technologies such as TMS are not considered epileptogenic. Current animal and clinical evidence does not support epilepsy generation with conventional tDCS protocols.

Beyond seizure initiation, a concern for epileptogenesis is a theoretical concern with any electrical brain stimulation technology. Electrical cortical, hippocampal or amygdala stimulation can produce epilepsy in animal models through "kindling" [150], [151], where cumulative effects of recurrent stimulation lower the seizure threshold. However, such effects have not been seen with tDCS. As above, conventional tDCS intensity is far below seizure induction threshold, and even much higher-intensity stimulation, as by TMS are not considered epileptogenic. Current animal and clinical evidence does not support epilepsy generation by conventional tDCS protocols. 


\section{tDCS Special Considerations for Safety in Stroke}

In studies of tDCS in persons with stroke, adults and children, published since 2014 ( $N=33$ studies), there are 2 studies reporting minor adverse events [79], [152] including mild headache, sleepiness, and various sensations. In addition, there are few reports of dropouts with 14 from 6 studies [153], [79], [28], [154], [155], [156] out of 507 total participants across 33 studies. Reporting criteria and reasons for dropouts vary and include personal reasons (e.g. unrelated medical problems, refusal to participate, etc.) that were not Serious Adverse Effects as defined here.

To date and to our knowledge, no persistent decrements in behavioral performance or mood have been documented as a result of tDCS application in stroke populations. Special consideration is required, however, regarding the methods used to determine whether a possible adverse behavioral or mood change was caused or aggravated by tDCS (see above discussion of [28]). The majority of stroke survivors commonly demonstrate one or more behavioral deficits (e.g., motor, sensory, perceptual, cognitive, speech-language, swallowing, etc.). Further, approximately $30 \%$ of stroke survivors report depression [157], [158], compared to $4-7.3 \%$ of the general adult population (Centers for Disease Control; CDC), and the presence and severity of depression may vary over time [159],[157]. Baseline assessment of deficits is clearly warranted, but stroke survivors often experience significant variability in performance for the affected behaviors from day to day, or even from morning to afternoon. Because of the presence of deficits, and the day-to-day behavioral variability, it would be possible to 
miscategorize a decrement in performance as a Serious Adverse Effect if adequate baseline data were not collected. To minimize this error, investigators may consider if multiple baseline data points on target behaviors as well as other comorbid behavioral/mood deficits should be gathered before tDCS administration. After tDCS, adverse behavioral or mood effects may be followed over time to document their duration.

When making inferences about the safety of tDCS based on the occurrence or nonoccurrence of Serious Adverse Effects in the studies to date, it is important to consider the population characteristics of the sample studied. The majority of tDCS studies in the stroke population so far have been conducted in relatively uncomplicated patients in the chronic phase after injury (beginning of the chronic phase of recovery vary from 2 months to 1 year post-stroke). Given the small but beneficial effects of tDCS on motor and language function in chronic patients, studies in acute (first days after stroke) to subacute (first 2-12 weeks after stroke) patients are also currently conducted with the intention to modulate recovery within an early plastic time window after injury. First published results indicate similar tolerability and safety of tDCS in acute and subacute patients compared to chronic stroke patients. However, this preliminary conclusion is deducted from studies with a small overall sample size.

Typically, stroke survivors with severe depression or other neuropsychiatric disorders, alcohol/drug abuse, history of seizure, or skull defects (typically resulting from brain surgery related to the stroke) have been excluded from tDCS studies. Additional data will 
thus be needed to extend the safety data on tDCS to stroke survivors with these comorbidities. tDCS studies in independent non-stroke samples with primary neuropsychiatric, substance abuse, and seizure disorders suggest that the risk of Serious Adverse Effects with these comorbidities is low (see above).

Up to $20 \%$ of stroke survivors will experience post-stroke seizures [160]. As noted above, there is no clear evidence that tDCS can induce seizures, even in cases of compromised neurophysiology. tDCS studies in stroke patients now include persons with history of seizures, although requiring a minimum period without seizure or changes to antiepileptic medications may be reasonable prior to administering tDCS as a proactive measure to avoid coincidental seizures.

A commonly stated concern is that the presence of skulls defects may increase risk by creating a "funnel" of current through the skull resulting in local concentrations of current density in the brain. Electrical field modeling suggests that typical tDCS protocols administered over a range of skull defects may result in local increase in current density compared to the intact-skull case, under worst-case theoretical conditions corresponding to a six-fold increase in brain current density [42], [161]. This does not exceed the current density threshold for injury reported in animal models. Increases in brain current density can be prevented or minimized by adjusting electrode montage. The exclusion of subjects from some human trials for this concern is not evidence for increased risk of a Serious Adverse Effect. 
Stroke survivors in some cases have abnormal cerebral vascular anatomy, reduced cerebral perfusion, and/or disrupted cerebrovascular autoregulation [162]. Though one study has demonstrated change in cerebrovascular autoregulation in controls in response to a single session of tDCS [163] (with one electrode positioned on the head and another on an arm), another recent study in a control population and in those with neurological abnormalities and/or cerebrovascular disease found no significant change in vasomotor reflex following a single session of bicephalic tDCS [164]. The lack of Serious Adverse Effects in tDCS studies of stroke to date suggests that any possible effect of tDCS on cerebral blood vessels are unlikely to cause clinically significant changes in brain perfusion. There is no evidence for increased risk in subjects with large vessel occlusions (e.g. complete blockage of an internal carotid artery) and such subjects are typically not a priori excluded from trials for safety reasons, though often they are excluded to ensure the homogeneity of the subject sample and some investigators suggest additional monitoring when included [164].

Data from animal models of brain injury were discussed in a previous section.

tDCS Special Consideration for Safety in Mood Disorders

Treatment-emergent (hypo)mania (TEM) is a potentially Serious Adverse Effect that can occur in depressed patients during pharmacologic antidepressant treatment, for instance, in up to $2.3 \%$ of patients with unipolar depression [63]. A variety of factors influence the likelihood of developing TEM, including the potency of the agent being used and the 
individual characteristics of the patient. There are four stand-alone case reports in literature [64], [65], [66], [67] and some reports in randomized clinical trials (1 case in [68] and 6 cases in [69], of which 5 patients were given tDCS combined with sertraline) of mania or hypomania induction after tDCS treatment. It is important to note that some of these patients were not known to have bipolar disorder. Most of these episodes resolved spontaneously when tDCS was withheld for a few days or with either small dose adjustments or introduction of a new pharmacotherapy. However one case was notable for a fulminant episode of mania with psychotic features [67]. In the other 5 patients who received sertraline combined with tDCS, it is not possible to disentangle with confidence which intervention (or both) was responsible for the symptoms and thus these were not Serious Adverse Effects per the definition of this review. Stimulation montage may be a factor, with one patient becoming hypomanic with a montage involving greater stimulation of deep central brain areas, but not with the usual frontal montage used to treat depression [66].

It is difficult to estimate the precise frequency of this adverse event (i.e., inducing manic/hypomanic episodes) or establish causality with tDCS application. As such, it is also unclear if having a diagnosis of bipolar disorder places a patient at theoretically higher risk of a manic switch with tDCS, as has been suggested for other brain stimulation therapies [70]. Conservatively one may adopt the same recommendations for tDCS in depressed patients as in antidepressant drug treatment, including careful observation of the patients' clinical outcomes while on a clinical treatment and careful assessment for a history of bipolar disorder or history of switching into mania with past 
antidepressant treatments (in these patients, concurrent treatment with mood stabilizer medications during the tDCS treatment course could be considered). But, consistent with the scope of this review, we emphasize a lack of convincing evidence that tDCS increases risk of manic switch. In trials for depression, greater than 4160 sessions have been applied across more than 430 subjects (Figure 7) without a documented Serious Adverse Effect.

While neurophysiological studies in healthy individuals have established a short-term interaction between tDCS and pharmacological agents [71], [72], [8], [73], there is minimal information on the interaction between tDCS and pharmacologic intervention in the treatment of mood disorders. The available evidence suggest potential synergistic effects at least in the treatment of depression [74],[75],[69]. There is no evidence that interactions between tDCS and pharmacological agents increase the risk of a Serious Adverse Effect.

tDCS Special Consideration for Subjects with Implants

For subjects with pre-existing implants, concerns about increased susceptibility to tDCS include 1) injury to the brain related to changes in brain current flow pattern as a result of a conductive implant and/or associated skull defect, that can lead to a local current concentration and 2) damage or disruption of device function, especially when the device includes an electrical sensing component (such as a pacemaker). The role of skull defects is addressed above, and modeling studies further do not support a significant increase in 
current concentration even under "worst case" conditions (Figure 8). Concern about interactions with pacemakers increases for extracephalic electrodes but remains hypothetical [165]. Though exclusion of subjects with preexisting implants, and especially head implants is common in tDCS trials as a precautionary measure [3], there is no evidence of injury to a subject with an implant and no theoretical risk of injury based on modeling. While metal inside the body has a high nominal conductivity, the metal is as an electron current carrier, whereas current carried by through the body (as produced by tDCS) is ionic; as such, a large electrode impedance is expected between the metal implant and the surrounding tissue making significant current flow across the metal implant unlikely [166]. Limited ongoing experience with tDCS in subjects with both DBS and cortical electrode arrays (the latter for pre-epilepsy surgical monitoring) suggests that stimulation is tolerated (Bikson, in preparation). Thus, while pre-existing implants remain a theoretical concern, neither theory nor limited clinical experience establish evidence for increased risk of Serious Adverse Effects.

tDCS Special Considerations for Safety in Home Use

With the advance towards the clinical use of tDCS for a wide range of applications, home use (as opposed to in-clinic administration) will presumably become more frequent. There is evidence that beneficial effects may be achieved with cumulative sessions [167], including when paired with a behavioral program to enhance outcome (e.g. cognitive or physical exercises for recovery of function, e.g., [168], [169], [170]). Therefore, repeated tDCS administration over time will likely be essential to the effectiveness of many 
treatments. In this sense, home use may also be helpful to either continue or sustain an initial therapeutic benefit, possibly spanning months (e.g. 100 sessions or more) [171], [61]. Alternatively, for other applications, tDCS may be utilized "on demand" for situational use (e.g. to increase or sustain attentional vigilance [172], [58]). These potential scenarios create an interest in clinical trials with remote use. Repeat and/or spontaneous visits to a clinic location are often not plausible due to the obstacles of time commitment as well as logistical and scheduling challenges. Dependence on in-clinic administration of tDCS would also increase the overall costs of treatment. This section focuses on home use where tDCS is either self-applied or applied by a local caregiver, as opposed to an investigator travelling to the patients home for every session, which reduces the burden on patients but not the investigators or cost.

While there has been no established Serious Adverse Effects for home-use (including self-directed) tDCS, several notes of caution have been raised. These are noted here, although we again emphasize that our approach is data-driven, rather than speculative [173]. Foremost, there are potential issues with the overall design and manufacture of the actual device and equipment, including the reliability of construction, consistency of electrode placement and contact quality, safety meters to prevent overuse (intentional or unintentional), and sanitary practices for the handling of sponges and electrodes. Furthermore, once implemented, there are concerns regarding the behavioral correlates of electrode placement [174] as well as the possibility of unexpected effects with improper dose [175]. Also, especially relevant for clinical conditions is the consideration that tDCS may potentially interact with other therapies or medications [176]. While all these 
concerns also apply for in-clinic administration, the long term and variable naturalistic setting of home-use, as well as issues surrounding self-directed stimulation, requires special attention.

There have been relatively few clinical trials to include the home use of tDCS. Using a crossover design, one study [177] employed home use of tDCS for the treatment of trigeminal neuralgia. Participants $(n=17)$ were instructed to self-apply tDCS at home over two 2-week periods separated by one month in which they applied active stimulation (1 $\mathrm{mA}$ ) and sham (in randomized order) over the primary motor cortex. Participants were provided with a battery-operated device used in conventional clinical trials and study staff were available by phone. Active stimulation was found to be effective for reducing pain, with no adverse events reported and overall good tolerability. The study had a relatively high dropout rate, with only 10 subjects completing both study conditions. Some participants had difficulty self-applying tDCS and the authors noted that further training would be useful.

The clinical study of tDCS with home use must be both safe and reliable, as well as measurable and reproducible. A group of clinical investigators interested in home use of tDCS developed a set of guidelines for home use in clinical trials, governed by remotesupervision through a telemedicine platform [178]. Central to these recommendations is specially-designed equipment that both carefully regulates and records use. Extensive training procedures and safety checks at each step governed by a study technician can 
guide safe self-application (or application by caregiver or other proxy) and ensure the safest and most tolerable use.

The first employment of these guidelines was used to develop a protocol for use in participants with multiple sclerosis (MS) [179]. The protocol centers on a speciallydesigned pre-programmed device that features a code to "unlock" delivery of only one stimulation (or sham) session at a time. The code is issued by a remote study technician over HIPAA-compliant videoconferencing, and is only provided after ensuring all safety checks are met. With this protocol, targeting 10 sessions over two weeks, 24 participants have completed 232 sessions without any adverse event or discontinuation of any session. There has been high tolerability and compliance.

A controlled and sequential plan of study to extend use away from the clinic setting and without the investigator/clinician present will provide the safest and best route for home use. As an extension of in-clinic tDCS applications, the continued integration of structured and supervised remote application in clinical trials is the optimal route for determining and addressing safety concerns for home use. A next step will be to provide participants in these trials with the option to continue use without real-time supervision, once they have been adequately trained and monitored to complete safe and effective use on their own. While this section adopts a cautious tone, there is no evidence of a serious adverse event in the limited controlled human trials of home-use tDCS. Rigorous deployment planning, which includes specially designed devices and guidance, is 
intended to maintain a flat level a risk in the transition from clinic to home; only in this case, can safety data from all clinical trials be used to directly support home-use [178].

Experience with so-called Do-It-Yourself (DIY) devices (which includes home-made electronics and electrodes) and with devices marketed directly to consumers (which have not been tested in controlled human trials) are not within the scope of this review [180], [173], [181]. Nor are the findings of this review necessarily relevant to these situations. Home-use discussed here is limited to the context of supervised human trials.

\section{Conclusions}

This review examined evidence on the safety of tDCS. Evidence for brain injury by DCS in animal models occurs at intensities over an order of magnitude above intensities used in conventional tDCS. To date, based on over a total 33,000 sessions and over 1,000 subjects who received repeated tDCS sessions, there is no evidence for irreversible injury produced by conventional tDCS protocols within a wide range of stimulation parameters ( $\leq 40 \mathrm{~min}, \leq 4 \mathrm{~mA}, \leq 7.2 \mathrm{C}$ ). This analysis consolidates and adds to existing evidence on tDCS safety and facilitates further research of tDCS in human subjects. These conclusions are in agreement with a prior analyses and review focused on single center experiences [55].

\section{COI:}


MB has equity in Soterix Medical Inc. The City University of New York has patents on brain stimulation with MB as inventor. MAN is member of the Advisory Board of Neuroelectrics. CL has received equipment support from Soterix Medical Inc.

\section{Acknowledgments:}

MB is supported by the NIH (Award\# 5R03EB017410-02 5R21EB017510-02

5R01MH092926-05) and DoD (Award\# FA9550-13-1-0073). AJW is supported by the McKnight Brain Research Foundation, the Center for Cognitive Aging and Memory at the University of Florida, and by the NIH/NCATS CTSA grant UL1 TR000064 and KL2 TR000065. 


\section{References:}

[1] Sawyer DW, Donowitz GR, Mandell GL. Polymorphonuclear neutrophils: an effective antimicrobial force. Rev Infect Dis 1989;11 Suppl 7:S1532-44.

[2] Nitsche MA, Niehaus L, Hoffmann KT, Hengst S, Liebetanz D, Paulus W, et al. MRI study of human brain exposed to weak direct current stimulation of the frontal cortex. Clin Neurophysiol Off J Int Fed Clin Neurophysiol 2004;115:2419_ 23. doi:10.1016/j.clinph.2004.05.001.

[3] Brunoni AR, Nitsche MA, Bolognini N, Bikson M, Wagner T, Merabet L, et al. Clinical research with transcranial direct current stimulation (tDCS): challenges and future directions. Brain Stimulat 2012;5:175-95. doi:10.1016/j.brs.2011.03.002.

[4] Medeiros LF, de Souza ICC, Vidor LP, de Souza A, Deitos A, Volz MS, et al. Neurobiological effects of transcranial direct current stimulation: a review. Front Psychiatry 2012;3:110. doi:10.3389/fpsyt.2012.00110.

[5] Kim SJ, Kim BK, Ko YJ, Bang MS, Kim MH, Han TR. Functional and histologic changes after repeated transcranial direct current stimulation in rat stroke model. J Korean Med Sci 2010;25:1499-505. doi:10.3346/jkms.2010.25.10.1499.

[6] Fregni F, Nitsche MA, Loo CK, Brunoni AR, Marangolo P, Leite J, et al. Regulatory Considerations for the Clinical and Research Use of Transcranial Direct Current Stimulation (tDCS): review and recommendations from an expert panel. Clin Res Regul Aff 2015;32:22-35. doi:10.3109/10601333.2015.980944.

[7] Peterchev AV, Wagner TA, Miranda PC, Nitsche MA, Paulus W, Lisanby SH, et al. Fundamentals of transcranial electric and magnetic stimulation dose: definition, selection, and reporting practices. Brain Stimulat 2012;5:435-53. doi:10.1016/j.brs.2011.10.001.

[8] Fresnoza S, Paulus W, Nitsche MA, Kuo M-F. Nonlinear dose-dependent impact of D1 receptor activation on motor cortex plasticity in humans. J Neurosci Off J Soc Neurosci 2014;34:2744-53. doi:10.1523/JNEUROSCI.3655-13.2014.

[9] Liebetanz D, Klinker F, Hering D, Koch R, Nitsche MA, Potschka H, et al. Anticonvulsant effects of transcranial direct-current stimulation (tDCS) in the rat cortical ramp model of focal epilepsy. Epilepsia 2006;47:1216-24. doi:10.1111/j.1528-1167.2006.00539.x.

[10] Batsikadze G, Moliadze V, Paulus W, Kuo M-F, Nitsche MA. Partially non-linear stimulation intensity-dependent effects of direct current stimulation on motor cortex excitability in humans. J Physiol 2013;591:1987-2000. doi:10.1113/jphysiol.2012.249730.

[11] Bikson M, Datta A, Rahman A, Scaturro J. Electrode montages for tDCS and weak transcranial electrical stimulation: role of "return" electrode's position and size. Clin Neurophysiol Off J Int Fed Clin Neurophysiol 2010;121:1976-8. doi:10.1016/j.clinph.2010.05.020.

[12] Datta A, Bansal V, Diaz J, Patel J, Reato D, Bikson M. Gyri-precise head model of transcranial direct current stimulation: improved spatial focality using a ring electrode versus conventional rectangular pad. Brain Stimulat 2009;2:201-7, 207.e1. doi:10.1016/j.brs.2009.03.005. 
[13] Domingo JL, Llobet JM, Corbella J. Toxic effects of vanadium in streptozotocintreated rats after administration of vanadate to normalize blood glucose levels. Diabetologia 1990;33:62-3.

[14] Kronberg G, Bikson M. Electrode assembly design for transcranial Direct Current Stimulation: a FEM modeling study. Conf Proc Annu Int Conf IEEE Eng Med Biol Soc IEEE Eng Med Biol Soc Annu Conf 2012;2012:891-5. doi:10.1109/EMBC.2012.6346075.

[15] Miranda PC, Faria P, Hallett M. What does the ratio of injected current to electrode area tell us about current density in the brain during tDCS? Clin Neurophysiol Off J Int Fed Clin Neurophysiol 2009;120:1183-7. doi:10.1016/j.clinph.2009.03.023.

[16] Nitsche MA, Bikson M, Bestmann S. On the Use of Meta-analysis in Neuromodulatory Non-invasive Brain Stimulation. Brain Stimulat 2015;8:666-7. doi:10.1016/j.brs.2015.03.008.

[17] Liebetanz D, Koch R, Mayenfels S, König F, Paulus W, Nitsche MA. Safety limits of cathodal transcranial direct current stimulation in rats. Clin Neurophysiol Off $\mathbf{J}$ Int Fed Clin Neurophysiol 2009;120:1161-7. doi:10.1016/j.clinph.2009.01.022.

[18] Sundaram A, Stock V, Cruciani RA, Knotkova H. Safety of transcranial direct current stimulation (tDCS) in protocols involving human subjects. J Pain Manag 2009;2:285-93.

[19] Nitsche MA, Paulus W. Excitability changes induced in the human motor cortex by weak transcranial direct current stimulation. J Physiol 2000;527 Pt 3:633-9.

[20] Shiozawa P, da Silva ME, Raza R, Uchida RR, Cordeiro Q, Fregni F, et al. Safety of repeated transcranial direct current stimulation in impaired skin: a case report. $\mathrm{J}$ ECT 2013;29:147-8. doi:10.1097/YCT.0b013e318279c1a1.

[21] DaSilva AF, Volz MS, Bikson M, Fregni F. Electrode positioning and montage in transcranial direct current stimulation. J Vis Exp JoVE 2011. doi:10.3791/2744.

[22] Nitsche MA, Doemkes S, Karaköse T, Antal A, Liebetanz D, Lang N, et al. Shaping the effects of transcranial direct current stimulation of the human motor cortex. J Neurophysiol 2007;97:3109-17. doi:10.1152/jn.01312.2006.

[23] Minhas P, Bansal V, Patel J, Ho JS, Diaz J, Datta A, et al. Electrodes for highdefinition transcutaneous DC stimulation for applications in drug delivery and electrotherapy, including tDCS. J Neurosci Methods 2010;190:188-97. doi:10.1016/j.jneumeth.2010.05.007.

[24] Dmochowski JP, Datta A, Huang Y, Richardson JD, Bikson M, Fridriksson J, et al. Targeted transcranial direct current stimulation for rehabilitation after stroke. NeuroImage 2013;75:12-9. doi:10.1016/j.neuroimage.2013.02.049.

[25] Dmochowski JP, Bikson M, Datta A, Richardson J, Fridriksson J, Parra LC. On the role of electric field orientation in optimal design of transcranial current stimulation. Conf Proc Annu Int Conf IEEE Eng Med Biol Soc IEEE Eng Med Biol Soc Annu Conf 2012;2012:6426-9. doi:10.1109/EMBC.2012.6347465.

[26] Dmochowski JP, Datta A, Bikson M, Su Y, Parra LC. Optimized multi-electrode stimulation increases focality and intensity at target. J Neural Eng 2011;8:046011. doi:10.1088/1741-2560/8/4/046011. 
[27] Pirulli C, Fertonani A, Miniussi C. Is neural hyperpolarization by cathodal stimulation always detrimental at the behavioral level? Front Behav Neurosci 2014;8:226. doi:10.3389/fnbeh.2014.00226.

[28] Smit M, Schutter DJLG, Nijboer TCW, Visser-Meily JMA, Kappelle LJ, Kant N, et al. Transcranial direct current stimulation to the parietal cortex in hemispatial neglect: A feasibility study. Neuropsychologia 2015;74:152-61. doi:10.1016/j.neuropsychologia.2015.04.014.

[29] Brunoni AR, Amadera J, Berbel B, Volz MS, Rizzerio BG, Fregni F. A systematic review on reporting and assessment of adverse effects associated with transcranial direct current stimulation. Int J Neuropsychopharmacol Off Sci J Coll Int Neuropsychopharmacol CINP 2011;14:1133-45. doi:10.1017/S1461145710001690.

[30] Guarienti F, Caumo W, Shiozawa P, Cordeiro Q, Boggio PS, Benseñor IM, et al. Reducing transcranial direct current stimulation-induced erythema with skin pretreatment: considerations for sham-controlled clinical trials. Neuromodulation J Int Neuromodulation Soc 2015;18:261-5. doi:10.1111/ner.12230.

[31] Mielke D, Wrede A, Schulz-Schaeffer W, Taghizadeh-Waghefi A, Nitsche MA, Rohde V, et al. Cathodal transcranial direct current stimulation induces regional, long-lasting reductions of cortical blood flow in rats. Neurol Res 2013;35:102937. doi:10.1179/1743132813Y.0000000248.

[32] Wachter D, Wrede A, Schulz-Schaeffer W, Taghizadeh-Waghefi A, Nitsche MA, Kutschenko A, et al. Transcranial direct current stimulation induces polarityspecific changes of cortical blood perfusion in the rat. Exp Neurol 2011;227:3227. doi:10.1016/j.expneurol.2010.12.005.

[33] Minhas P, Datta A, Bikson M. Cutaneous perception during tDCS: role of electrode shape and sponge salinity. Clin Neurophysiol Off J Int Fed Clin Neurophysiol 2011;122:637-8. doi:10.1016/j.clinph.2010.09.023.

[34] Rodríguez N, Opisso E, Pascual-Leone A, Soler MD. Skin lesions induced by transcranial direct current stimulation (tDCS). Brain Stimulat 2014;7:765-7. doi:10.1016/j.brs.2014.06.005.

[35] Palm U, Feichtner KB, Hasan A, Gauglitz G, Langguth B, Nitsche MA, et al. The role of contact media at the skin-electrode interface during transcranial direct current stimulation (tDCS). Brain Stimulat 2014;7:762-4. doi:10.1016/j.brs.2014.06.006.

[36] Wang J, Wei Y, Wen J, Li X. Skin burn after single session of transcranial direct current stimulation (tDCS). Brain Stimulat 2015;8:165-6. doi:10.1016/j.brs.2014.10.015.

[37] Frank E, Wilfurth S, Landgrebe M, Eichhammer P, Hajak G, Langguth B. Anodal skin lesions after treatment with transcranial direct current stimulation. Brain Stimulat 2010;3:58-9. doi:10.1016/j.brs.2009.04.002.

[38] Bikson M, Datta A, Elwassif M. Establishing safety limits for transcranial direct current stimulation. Clin Neurophysiol Off J Int Fed Clin Neurophysiol 2009;120:1033-4. doi:10.1016/j.clinph.2009.03.018.

[39] Woods AJ, Antal A, Bikson M, Boggio PS, Brunoni AR, Celnik P, et al. A technical guide to tDCS, and related non-invasive brain stimulation tools. Clin 
Neurophysiol Off J Int Fed Clin Neurophysiol 2015.

doi:10.1016/j.clinph.2015.11.012.

[40] Edwards D, Cortes M, Datta A, Minhas P, Wassermann EM, Bikson M. Physiological and modeling evidence for focal transcranial electrical brain stimulation in humans: a basis for high-definition tDCS. NeuroImage 2013;74:266-75. doi:10.1016/j.neuroimage.2013.01.042.

[41] Datta A, Baker JM, Bikson M, Fridriksson J. Individualized model predicts brain current flow during transcranial direct-current stimulation treatment in responsive stroke patient. Brain Stimulat 2011;4:169-74. doi:10.1016/j.brs.2010.11.001.

[42] Datta A, Bikson M, Fregni F. Transcranial direct current stimulation in patients with skull defects and skull plates: high-resolution computational FEM study of factors altering cortical current flow. NeuroImage 2010;52:1268-78. doi:10.1016/j.neuroimage.2010.04.252.

[43] Gillick BT, Kirton A, Carmel JB, Minhas P, Bikson M. Pediatric stroke and transcranial direct current stimulation: methods for rational individualized dose optimization. Front Hum Neurosci 2014;8:739. doi:10.3389/fnhum.2014.00739.

[44] Kessler SK, Minhas P, Woods AJ, Rosen A, Gorman C, Bikson M. Dosage considerations for transcranial direct current stimulation in children: a computational modeling study. PloS One 2013;8:e76112. doi:10.1371/journal.pone.0076112.

[45] Mekonnen A, Salvador R, Ruffini G, Miranda PC. The relationship between transcranial current stimulation electrode montages and the effect of the skull orbital openings. Conf Proc Annu Int Conf IEEE Eng Med Biol Soc IEEE Eng Med Biol Soc Annu Conf 2012;2012:831-4. doi:10.1109/EMBC.2012.6346060.

[46] Laakso I, Hirata A. Computational analysis shows why transcranial alternating current stimulation induces retinal phosphenes. J Neural Eng 2013;10:046009. doi:10.1088/1741-2560/10/4/046009.

[47] Sadleir RJ, Vannorsdall TD, Schretlen DJ, Gordon B. Transcranial direct current stimulation (tDCS) in a realistic head model. NeuroImage 2010;51:1310-8. doi:10.1016/j.neuroimage.2010.03.052.

[48] Rampersad SM, Janssen AM, Lucka F, Aydin Ü, Lanfer B, Lew S, et al. Simulating transcranial direct current stimulation with a detailed anisotropic human head model. IEEE Trans Neural Syst Rehabil Eng Publ IEEE Eng Med Biol Soc 2014;22:441-52. doi:10.1109/TNSRE.2014.2308997.

[49] Mattai A, Miller R, Weisinger B, Greenstein D, Bakalar J, Tossell J, et al. Tolerability of transcranial direct current stimulation in childhood-onset schizophrenia. Brain Stimulat 2011;4:275-80. doi:10.1016/j.brs.2011.01.001.

[50] Grecco LAC, Duarte NAC, Zanon N, Galli M, Fregni F, Oliveira CS. Effect of a single session of transcranial direct-current stimulation on balance and spatiotemporal gait variables in children with cerebral palsy: A randomized shamcontrolled study. Braz J Phys Ther 2014;18:419-27.

[51] Grecco LAC, de Almeida Carvalho Duarte N, Mendonça ME, Cimolin V, Galli M, Fregni F, et al. Transcranial direct current stimulation during treadmill training in children with cerebral palsy: a randomized controlled double-blind clinical trial. Res Dev Disabil 2014;35:2840-8. doi:10.1016/j.ridd.2014.07.030. 
[52] Vestito L, Rosellini S, Mantero M, Bandini F. Long-term effects of transcranial direct-current stimulation in chronic post-stroke aphasia: a pilot study. Front Hum Neurosci 2014;8:785. doi:10.3389/fnhum.2014.00785.

[53] Kessler SK, Turkeltaub PE, Benson JG, Hamilton RH. Differences in the experience of active and sham transcranial direct current stimulation. Brain Stimulat 2012;5:155-62. doi:10.1016/j.brs.2011.02.007.

[54] Morales-Quezada L, Cosmo C, Carvalho S, Leite J, Castillo-Saavedra L, Rozisky $\mathrm{JR}$, et al. Cognitive effects and autonomic responses to transcranial pulsed current stimulation. Exp Brain Res 2015;233:701-9. doi:10.1007/s00221-014-4147-y.

[55] Poreisz C, Boros K, Antal A, Paulus W. Safety aspects of transcranial direct current stimulation concerning healthy subjects and patients. Brain Res Bull 2007;72:208-14. doi:10.1016/j.brainresbull.2007.01.004.

[56] Raimundo RJS, Uribe CE, Brasil-Neto JP. Lack of clinically detectable acute changes on autonomic or thermoregulatory functions in healthy subjects after transcranial direct current stimulation (tDCS). Brain Stimulat 2012;5:196-200. doi:10.1016/j.brs.2011.03.009.

[57] Nitsche MA, Liebetanz D, Lang N, Antal A, Tergau F, Paulus W. Safety criteria for transcranial direct current stimulation (tDCS) in humans. Clin Neurophysiol Off J Int Fed Clin Neurophysiol 2003;114:2220-2; author reply 2222-3.

[58] McIntire LK, McKinley RA, Goodyear C, Nelson J. A comparison of the effects of transcranial direct current stimulation and caffeine on vigilance and cognitive performance during extended wakefulness. Brain Stimulat 2014;7:499-507. doi:10.1016/j.brs.2014.04.008.

[59] Tadini L, El-Nazer R, Brunoni AR, Williams J, Carvas M, Boggio P, et al. Cognitive, mood, and electroencephalographic effects of noninvasive cortical stimulation with weak electrical currents. J ECT 2011;27:134-40. doi:10.1097/YCT.0b013e3181e631a8.

[60] Russo R, Wallace D, Fitzgerald PB, Cooper NR. Perception of comfort during active and sham transcranial direct current stimulation: a double blind study. Brain Stimulat 2013;6:946-51. doi:10.1016/j.brs.2013.05.009.

[61] Andrade C. Once- to twice-daily, 3-year domiciliary maintenance transcranial direct current stimulation for severe, disabling, clozapine-refractory continuous auditory hallucinations in schizophrenia. J ECT 2013;29:239-42. doi:10.1097/YCT.0b013e3182843866.

[62] Paneri B, Khadka N, Patel V, Thomas C, Tyler W, Parra LC, et al. The tolerability of transcranial electrical stimulation used across extended periods in a naturalistic context by healthy individuals. PeerJ Prepr 2015;3.

[63] Benvenuti A, Rucci P, Miniati M, Papasogli A, Fagiolini A, Cassano GB, et al. Treatment-emergent mania/hypomania in unipolar patients. Bipolar Disord 2008;10:726-32. doi:10.1111/j.1399-5618.2008.00613.x.

[64] Arul-Anandam AP, Loo C, Mitchell P. Induction of hypomanic episode with transcranial direct current stimulation. J ECT 2010;26:68-9. doi:10.1097/YCT.0b013e3181a744bf.

[65] Baccaro A, Brunoni AR, Bensenor IM, Fregni F. Hypomanic episode in unipolar depression during transcranial direct current stimulation. Acta Neuropsychiatr 2010;22:316-8. 
[66] Gálvez V, Alonzo A, Martin D, Mitchell PB, Sachdev P, Loo CK. Hypomania induction in a patient with bipolar II disorder by transcranial direct current stimulation (tDCS). J ECT 2011;27:256-8. doi:10.1097/YCT.0b013e3182012b89.

[67] Brunoni AR, Valiengo L, Zanao T, de Oliveira JF, Bensenor IM, Fregni F. Manic psychosis after sertraline and transcranial direct-current stimulation. $\mathbf{J}$ Neuropsychiatry Clin Neurosci 2011;23:E4-5. doi:10.1176/jnp.23.3.jnpe4.

[68] Loo CK, Alonzo A, Martin D, Mitchell PB, Galvez V, Sachdev P. Transcranial direct current stimulation for depression: 3-week, randomised, sham-controlled trial. Br J Psychiatry J Ment Sci 2012;200:52-9. doi:10.1192/bjp.bp.111.097634.

[69] Brunoni AR, Valiengo L, Baccaro A, Zanão TA, de Oliveira JF, Goulart A, et al. The sertraline vs. electrical current therapy for treating depression clinical study: results from a factorial, randomized, controlled trial. JAMA Psychiatry 2013;70:383-91. doi:10.1001/2013.jamapsychiatry.32.

[70] Loo C, Katalinic N, Mitchell PB, Greenberg B. Physical treatments for bipolar disorder: a review of electroconvulsive therapy, stereotactic surgery and other brain stimulation techniques. J Affect Disord 2011;132:1-13. doi:10.1016/j.jad.2010.08.017.

[71] Lugon MDMV, Batsikadze G, Fresnoza S, Grundey J, Kuo M-F, Paulus W, et al. Mechanisms of Nicotinic Modulation of Glutamatergic Neuroplasticity in Humans. Cereb Cortex N Y N 1991 2015. doi:10.1093/cercor/bhv252.

[72] Fresnoza S, Stiksrud E, Klinker F, Liebetanz D, Paulus W, Kuo M-F, et al. Dosage-dependent effect of dopamine D2 receptor activation on motor cortex plasticity in humans. J Neurosci Off J Soc Neurosci 2014;34:10701-9. doi:10.1523/JNEUROSCI.0832-14.2014.

[73] Batsikadze G, Paulus W, Kuo M-F, Nitsche MA. Effect of serotonin on paired associative stimulation-induced plasticity in the human motor cortex. Neuropsychopharmacol Off Publ Am Coll Neuropsychopharmacol 2013;38:2260 7. doi:10.1038/npp.2013.127.

[74] Kuo H-I, Paulus W, Batsikadze G, Jamil A, Kuo M-F, Nitsche MA. Chronic Enhancement of Serotonin Facilitates Excitatory Transcranial Direct Current Stimulation-Induced Neuroplasticity. Neuropsychopharmacol Off Publ Am Coll Neuropsychopharmacol 2015. doi:10.1038/npp.2015.270.

[75] Shiozawa P, Cordeiro Q, Fregni F, Brunoni AR. Is sertraline plus transcranial direct current stimulation the future of effective depression treatment? J Comp Eff Res 2013;2:213-5. doi:10.2217/cer.13.28.

[76] San-Juan D, Calcáneo J de DDC, González-Aragón MF, Bermúdez Maldonado L, Avellán AM, Argumosa EVG, et al. Transcranial direct current stimulation in adolescent and adult Rasmussen's encephalitis. Epilepsy Behav EB 2011;20:12631. doi:10.1016/j.yebeh.2010.10.031.

[77] Cosmo C, Baptista AF, de Sena EP. Contribution of transcranial direct current stimulation on inhibitory control to assess the neurobiological aspects of attention deficit hyperactivity disorder: randomized controlled trial. JMIR Res Protoc 2015;4:e56. doi:10.2196/resprot.4138.

[78] Auvichayapat N, Rotenberg A, Gersner R, Ngodklang S, Tiamkao S, Tassaneeyakul W, et al. Transcranial direct current stimulation for treatment of 
refractory childhood focal epilepsy. Brain Stimulat 2013;6:696-700. doi:10.1016/j.brs.2013.01.009.

[79] Gillick BT, Feyma T, Menk J, Usset M, Vaith A, Wood TJ, et al. Safety and feasibility of transcranial direct current stimulation in pediatric hemiparesis: randomized controlled preliminary study. Phys Ther 2015;95:337-49. doi:10.2522/ptj.20130565.

[80] Young SJ, Bertucco M, Sheehan-Stross R, Sanger TD. Cathodal transcranial direct current stimulation in children with dystonia: a pilot open-label trial. J Child Neurol 2013;28:1238-44. doi:10.1177/0883073812460092.

[81] Duarte N de AC, Grecco LAC, Galli M, Fregni F, Oliveira CS. Effect of transcranial direct-current stimulation combined with treadmill training on balance and functional performance in children with cerebral palsy: a double-blind randomized controlled trial. PloS One 2014;9:e105777. doi:10.1371/journal.pone.0105777.

[82] Grecco LAC, E Mendonça M, Duarte NAC, Zanon N, Fregni F, Oliveira CS. Transcranial Direct Current Stimulation Combined with Treadmill Gait Training in Delayed Neuro-psychomotor Development. J Phys Ther Sci 2014;26:945-50. doi:10.1589/jpts.26.945.

[83] Aree-uea B, Auvichayapat N, Janyacharoen T, Siritaratiwat W, Amatachaya A, Prasertnoo J, et al. Reduction of spasticity in cerebral palsy by anodal transcranial direct current stimulation. J Med Assoc Thail Chotmaihet Thangphaet 2014;97:954-62.

[84] Walbert T, Chasteen K. Palliative and supportive care for glioma patients. Cancer Treat Res 2015;163:171-84. doi:10.1007/978-3-319-12048-5_11.

[85] Young SJ, Bertucco M, Sanger TD. Cathodal transcranial direct current stimulation in children with dystonia: a sham-controlled study. J Child Neurol 2014;29:232-9. doi:10.1177/0883073813492385.

[86] Bhanpuri NH, Bertucco M, Young SJ, Lee AA, Sanger TD. Multiday Transcranial Direct Current Stimulation Causes Clinically Insignificant Changes in Childhood Dystonia: A Pilot Study. J Child Neurol 2015;30:1604-15. doi: $10.1177 / 0883073815575369$.

[87] Anton SD, Woods AJ, Ashizawa T, Barb D, Buford TW, Carter CS, et al. Successful aging: Advancing the science of physical independence in older adults. Ageing Res Rev 2015;24:304-27. doi:10.1016/j.arr.2015.09.005.

[88] Salthouse TA. Selective review of cognitive aging. J Int Neuropsychol Soc 2010;16:754-60.

[89] Stephen LJ, Brodie MJ. Epilepsy in elderly people. Lancet Lond Engl 2000;355:1441-6. doi:10.1016/S0140-6736(00)02149-8.

[90] Hsu W-Y, Ku Y, Zanto TP, Gazzaley A. Effects of noninvasive brain stimulation on cognitive function in healthy aging and Alzheimer's disease: a systematic review and meta-analysis. Neurobiol Aging 2015;36:2348-59. doi:10.1016/j.neurobiolaging.2015.04.016.

[91] Berryhill ME, Jones KT. tDCS selectively improves working memory in older adults with more education. Neurosci Lett 2012;521:148-51. doi:10.1016/j.neulet.2012.05.074. 
[92] Boggio PS, Campanhã C, Valasek CA, Fecteau S, Pascual-Leone A, Fregni F. Modulation of decision-making in a gambling task in older adults with transcranial direct current stimulation. Eur J Neurosci 2010;31:593-7. doi:10.1111/j.14609568.2010.07080.x.

[93] Fertonani A, Brambilla M, Cotelli M, Miniussi C. The timing of cognitive plasticity in physiological aging: a tDCS study of naming. Front Aging Neurosci 2014;6:131. doi:10.3389/fnagi.2014.00131.

[94] Flöel A, Suttorp W, Kohl O, Kürten J, Lohmann H, Breitenstein C, et al. Noninvasive brain stimulation improves object-location learning in the elderly. Neurobiol Aging 2012;33:1682-9. doi:10.1016/j.neurobiolaging.2011.05.007.

[95] Goodwill AM, Reynolds J, Daly RM, Kidgell DJ. Formation of cortical plasticity in older adults following tDCS and motor training. Front Aging Neurosci 2013;5:87. doi:10.3389/fnagi.2013.00087.

[96] Hardwick RM, Celnik PA. Cerebellar direct current stimulation enhances motor learning in older adults. Neurobiol Aging 2014;35:2217-21. doi:10.1016/j.neurobiolaging.2014.03.030.

[97] Harty S, Robertson IH, Miniussi C, Sheehy OC, Devine CA, McCreery S, et al. Transcranial direct current stimulation over right dorsolateral prefrontal cortex enhances error awareness in older age. J Neurosci Off J Soc Neurosci 2014;34:3646-52. doi:10.1523/JNEUROSCI.5308-13.2014.

[98] Holland R, Leff AP, Josephs O, Galea JM, Desikan M, Price CJ, et al. Speech facilitation by left inferior frontal cortex stimulation. Curr Biol CB 2011;21:14037. doi:10.1016/j.cub.2011.07.021.

[99] Manenti R, Brambilla M, Petesi M, Ferrari C, Cotelli M. Enhancing verbal episodic memory in older and young subjects after non-invasive brain stimulation. Front Aging Neurosci 2013;5:49. doi:10.3389/fnagi.2013.00049.

[100] Meinzer M, Lindenberg R, Antonenko D, Flaisch T, Flöel A. Anodal transcranial direct current stimulation temporarily reverses age-associated cognitive decline and functional brain activity changes. J Neurosci Off J Soc Neurosci 2013;33:12470-8. doi:10.1523/JNEUROSCI.5743-12.2013.

[101] Meinzer M, Lindenberg R, Sieg MM, Nachtigall L, Ulm L, Flöel A. Transcranial direct current stimulation of the primary motor cortex improves word-retrieval in older adults. Front Aging Neurosci 2014;6:253. doi:10.3389/fnagi.2014.00253.

[102] Parikh PJ, Cole KJ. Effects of transcranial direct current stimulation on the control of finger force during dexterous manipulation in healthy older adults. PloS One 2015;10:e0124137. doi:10.1371/journal.pone.0124137.

[103] Park S-H, Seo J-H, Kim Y-H, Ko M-H. Long-term effects of transcranial direct current stimulation combined with computer-assisted cognitive training in healthy older adults. Neuroreport 2014;25:122-6. doi:10.1097/WNR.0000000000000080.

[104] Puri R, Hinder MR, Fujiyama H, Gomez R, Carson RG, Summers JJ. Durationdependent effects of the BDNF Val66Met polymorphism on anodal tDCS induced motor cortex plasticity in older adults: a group and individual perspective. Front Aging Neurosci 2015;7:107. doi:10.3389/fnagi.2015.00107.

[105] Ross LA, McCoy D, Coslett HB, Olson IR, Wolk DA. Improved proper name recall in aging after electrical stimulation of the anterior temporal lobes. Front Aging Neurosci 2011;3:16. doi:10.3389/fnagi.2011.00016. 
[106] Sandrini M, Brambilla M, Manenti R, Rosini S, Cohen LG, Cotelli M. Noninvasive stimulation of prefrontal cortex strengthens existing episodic memories and reduces forgetting in the elderly. Front Aging Neurosci 2014;6:289. doi:10.3389/fnagi.2014.00289.

[107] Zhou D, Zhou J, Chen H, Manor B, Lin J, Zhang J. Effects of transcranial direct current stimulation (tDCS) on multiscale complexity of dual-task postural control in older adults. Exp Brain Res 2015;233:2401-9. doi:10.1007/s00221-015-4310-0.

[108] Jones KT, Stephens JA, Alam M, Bikson M, Berryhill ME. Longitudinal neurostimulation in older adults improves working memory. PloS One 2015;10:e0121904. doi:10.1371/journal.pone.0121904.

[109] Manenti R, Brambilla M, Rosini S, Orizio I, Ferrari C, Borroni B, et al. Time up and go task performance improves after transcranial direct current stimulation in patient affected by Parkinson's disease. Neurosci Lett 2014;580:74-7. doi:10.1016/j.neulet.2014.07.052.

[110] Elder GJ, Taylor J-P. Transcranial magnetic stimulation and transcranial direct current stimulation: treatments for cognitive and neuropsychiatric symptoms in the neurodegenerative dementias? Alzheimers Res Ther 2014;6:74. doi:10.1186/s13195-014-0074-1.

[111] Khedr EM, Gamal NFE, El-Fetoh NA, Khalifa H, Ahmed EM, Ali AM, et al. A double-blind randomized clinical trial on the efficacy of cortical direct current stimulation for the treatment of Alzheimer's disease. Front Aging Neurosci 2014;6:275. doi:10.3389/fnagi.2014.00275.

[112] Boggio PS, Ferrucci R, Mameli F, Martins D, Martins O, Vergari M, et al. Prolonged visual memory enhancement after direct current stimulation in Alzheimer's disease. Brain Stimulat 2012;5:223-30. doi:10.1016/j.brs.2011.06.006.

[113] Boggio PS, Khoury LP, Martins DCS, Martins OEMS, de Macedo EC, Fregni F. Temporal cortex direct current stimulation enhances performance on a visual recognition memory task in Alzheimer disease. J Neurol Neurosurg Psychiatry 2009;80:444-7. doi:10.1136/jnnp.2007.141853.

[114] Cotelli M, Manenti R, Brambilla M, Petesi M, Rosini S, Ferrari C, et al. Anodal tDCS during face-name associations memory training in Alzheimer's patients. Front Aging Neurosci 2014;6:38. doi:10.3389/fnagi.2014.00038.

[115] Ferrucci R, Mameli F, Guidi I, Mrakic-Sposta S, Vergari M, Marceglia S, et al. Transcranial direct current stimulation improves recognition memory in Alzheimer disease. Neurology 2008;71:493-8. doi:10.1212/01.wnl.0000317060.43722.a3.

[116] Suemoto CK, Apolinario D, Nakamura-Palacios EM, Lopes L, Leite REP, Sales $\mathrm{MC}$, et al. Effects of a non-focal plasticity protocol on apathy in moderate Alzheimer's disease: a randomized, double-blind, sham-controlled trial. Brain Stimulat 2014;7:308-13. doi:10.1016/j.brs.2013.10.003.

[117] Benninger DH, Lomarev M, Lopez G, Wassermann EM, Li X, Considine E, et al. Transcranial direct current stimulation for the treatment of Parkinson's disease. J Neurol Neurosurg Psychiatry 2010;81:1105-11. doi:10.1136/jnnp.2009.202556.

[118] Boggio PS, Ferrucci R, Rigonatti SP, Covre P, Nitsche M, Pascual-Leone A, et al. Effects of transcranial direct current stimulation on working memory in patients 
with Parkinson's disease. J Neurol Sci 2006;249:31-8.

doi:10.1016/j.jns.2006.05.062.

[119] Doruk D, Gray Z, Bravo GL, Pascual-Leone A, Fregni F. Effects of tDCS on executive function in Parkinson's disease. Neurosci Lett 2014;582:27-31. doi:10.1016/j.neulet.2014.08.043.

[120] Fregni F, Boggio PS, Santos MC, Lima M, Vieira AL, Rigonatti SP, et al. Noninvasive cortical stimulation with transcranial direct current stimulation in Parkinson's disease. Mov Disord Off J Mov Disord Soc 2006;21:1693-702. doi:10.1002/mds.21012.

[121] Kaski D, Dominguez RO, Allum JH, Islam AF, Bronstein AM. Combining physical training with transcranial direct current stimulation to improve gait in Parkinson's disease: a pilot randomized controlled study. Clin Rehabil 2014;28:1115-24. doi:10.1177/0269215514534277.

[122] Elder GJ, Firbank MJ, Kumar H, Chatterjee P, Chakraborty T, Dutt A, et al. Effects of transcranial direct current stimulation upon attention and visuoperceptual function in Lewy body dementia: a preliminary study. Int Psychogeriatr IPA 2015:1-7. doi:10.1017/S1041610215001180.

[123] Manenti R, Bianchi M, Cosseddu M, Brambilla M, Rizzetti C, Padovani A, et al. Anodal transcranial direct current stimulation of parietal cortex enhances action naming in Corticobasal Syndrome. Front Aging Neurosci 2015;7:49. doi:10.3389/fnagi.2015.00049.

[124] Cotelli M, Manenti R, Petesi M, Brambilla M, Cosseddu M, Zanetti O, et al. Treatment of primary progressive aphasias by transcranial direct current stimulation combined with language training. J Alzheimers Dis JAD 2014;39:799808. doi:10.3233/JAD-131427.

[125] Gálvez V, Ho K-A, Alonzo A, Martin D, George D, Loo CK. Neuromodulation therapies for geriatric depression. Curr Psychiatry Rep 2015;17:59. doi:10.1007/s11920-015-0592-y.

[126] Yamamoto J, Ikeda A, Kinoshita M, Matsumoto R, Satow T, Takeshita K, et al. Low-frequency electric cortical stimulation decreases interictal and ictal activity in human epilepsy. Seizure 2006;15:520-7. doi:10.1016/j.seizure.2006.06.004.

[127] Swartzwelder HS, Lewis DV, Anderson WW, Wilson WA. Seizure-like events in brain slices: suppression by interictal activity. Brain Res 1987;410:362-6.

[128] Bikson M, Ghai RS, Baraban SC, Durand DM. Modulation of burst frequency, duration, and amplitude in the zero- $\mathrm{Ca}(2+)$ model of epileptiform activity. $\mathrm{J}$ Neurophysiol 1999;82:2262-70.

[129] Bikson M, Inoue M, Akiyama H, Deans JK, Fox JE, Miyakawa H, et al. Effects of uniform extracellular DC electric fields on excitability in rat hippocampal slices in vitro. J Physiol 2004;557:175-90. doi:10.1113/jphysiol.2003.055772.

[130] Kabakov AY, Muller PA, Pascual-Leone A, Jensen FE, Rotenberg A. Contribution of axonal orientation to pathway-dependent modulation of excitatory transmission by direct current stimulation in isolated rat hippocampus. J Neurophysiol 2012;107:1881-9. doi:10.1152/jn.00715.2011.

[131] Sunderam S, Gluckman B, Reato D, Bikson M. Toward rational design of electrical stimulation strategies for epilepsy control. Epilepsy Behav EB 2010;17:6-22. doi:10.1016/j.yebeh.2009.10.017. 
[132] Durand DM, Bikson M. Suppression and control of epileptiform activity by electrical stimulation: a review. Proc IEEE 2001;89:1065-82. doi:10.1109/5.939821.

[133] Reato D, Gasca F, Datta A, Bikson M, Marshall L, Parra LC. Transcranial electrical stimulation accelerates human sleep homeostasis. PLoS Comput Biol 2013;9:e1002898. doi:10.1371/journal.pcbi.1002898.

[134] Kamida T, Kong S, Eshima N, Fujiki M. Cathodal transcranial direct current stimulation affects seizures and cognition in fully amygdala-kindled rats. Neurol Res 2013;35:602-7. doi:10.1179/1743132813Y.0000000170.

[135] Kamida T, Kong S, Eshima N, Abe T, Fujiki M, Kobayashi H. Transcranial direct current stimulation decreases convulsions and spatial memory deficits following pilocarpine-induced status epilepticus in immature rats. Behav Brain Res 2011;217:99-103. doi:10.1016/j.bbr.2010.08.050.

[136] Dhamne SC, Ekstein D, Zhuo Z, Gersner R, Zurakowski D, Loddenkemper T, et al. Acute seizure suppression by transcranial direct current stimulation in rats. Ann Clin Transl Neurol 2015;2:843-56. doi:10.1002/acn3.226.

[137] San-Juan D, Morales-Quezada L, Orozco Garduño AJ, Alonso-Vanegas M, González-Aragón MF, Espinoza López DA, et al. Transcranial Direct Current Stimulation in Epilepsy. Brain Stimulat 2015;8:455-64. doi:10.1016/j.brs.2015.01.001.

[138] Fregni F, Thome-Souza S, Nitsche MA, Freedman SD, Valente KD, PascualLeone A. A controlled clinical trial of cathodal DC polarization in patients with refractory epilepsy. Epilepsia 2006;47:335-42. doi:10.1111/j.15281167.2006.00426.x.

[139] Yook S-W, Park S-H, Seo J-H, Kim S-J, Ko M-H. Suppression of seizure by cathodal transcranial direct current stimulation in an epileptic patient - a case report -. Ann Rehabil Med 2011;35:579-82. doi:10.5535/arm.2011.35.4.579.

[140] San-Juan D, Mayorga APM, Anschel DJ, Avellán AM, González-Aragón MF, Cole AJ. The double generalization phenomenon in juvenile absence epilepsy. Epilepsy Behav EB 2011;21:318-20. doi:10.1016/j.yebeh.2011.04.010.

[141] Faria P, Fregni F, Sebastião F, Dias AI, Leal A. Feasibility of focal transcranial DC polarization with simultaneous EEG recording: preliminary assessment in healthy subjects and human epilepsy. Epilepsy Behav EB 2012;25:417-25. doi:10.1016/j.yebeh.2012.06.027.

[142] Liu A, Bryant A, Jefferson A, Friedman D, Minhas P, Barnard S, et al. Exploring the efficacy of a 5-day course of transcranial direct current stimulation (TDCS) on depression and memory function in patients with well-controlled temporal lobe epilepsy. Epilepsy Behav EB 2015;55:11-20. doi:10.1016/j.yebeh.2015.10.032.

[143] Ekici B. Transcranial direct current stimulation-induced seizure: analysis of a case. Clin EEG Neurosci 2015;46:169. doi:10.1177/1550059414540647.

[144] Moliadze V, Schmanke T, Andreas S, Lyzhko E, Freitag CM, Siniatchkin M. Stimulation intensities of transcranial direct current stimulation have to be adjusted in children and adolescents. Clin Neurophysiol Off J Int Fed Clin Neurophysiol 2015;126:1392-9. doi:10.1016/j.clinph.2014.10.142.

[145] Rossi S, Hallett M, Rossini PM, Pascual-Leone A, Safety of TMS Consensus Group. Safety, ethical considerations, and application guidelines for the use of 
transcranial magnetic stimulation in clinical practice and research. Clin Neurophysiol Off J Int Fed Clin Neurophysiol 2009;120:2008-39. doi:10.1016/j.clinph.2009.08.016.

[146] Jennum P, Winkel H, Fuglsang-Frederiksen A, Dam M. EEG changes following repetitive transcranial magnetic stimulation in patients with temporal lobe epilepsy. Epilepsy Res 1994;18:167-73.

[147] Deng Z-D, Lisanby SH, Peterchev AV. Electric field depth-focality tradeoff in transcranial magnetic stimulation: simulation comparison of 50 coil designs. Brain Stimulat 2013;6:1-13. doi:10.1016/j.brs.2012.02.005.

[148] Tor P-C, Bautovich A, Wang M-J, Martin D, Harvey SB, Loo C. A systematic review and meta-analysis of brief versus ultrabrief right unilateral electroconvulsive therapy for depression. J Clin Psychiatry 2015. doi:10.4088/JCP.14r09145.

[149] Di Lazzaro V, Rothwell JC. Corticospinal activity evoked and modulated by noninvasive stimulation of the intact human motor cortex. J Physiol 2014;592:411528. doi:10.1113/jphysiol.2014.274316.

[150] Kandratavicius L, Balista PA, Lopes-Aguiar C, Ruggiero RN, Umeoka EH, Garcia-Cairasco N, et al. Animal models of epilepsy: use and limitations. Neuropsychiatr Dis Treat 2014;10:1693-705. doi:10.2147/NDT.S50371.

[151] McIntyre DC, Nathanson D, Edson N. A new model of partial status epilepticus based on kindling. Brain Res 1982;250:53-63.

[152] Kim YJ, Ku J, Cho S, Kim HJ, Cho YK, Lim T, et al. Facilitation of corticospinal excitability by virtual reality exercise following anodal transcranial direct current stimulation in healthy volunteers and subacute stroke subjects. J Neuroengineering Rehabil 2014;11:124. doi:10.1186/1743-0003-11-124.

[153] Cho H-S, Cha H-G. Effect of mirror therapy with tDCS on functional recovery of the upper extremity of stroke patients. J Phys Ther Sci 2015;27:1045-7. doi:10.1589/jpts.27.1045.

[154] Wu D, Wang J, Yuan Y. Effects of transcranial direct current stimulation on naming and cortical excitability in stroke patients with aphasia. Neurosci Lett 2015;589:115-20. doi:10.1016/j.neulet.2015.01.045.

[155] Fusco A, Assenza F, Iosa M, Izzo S, Altavilla R, Paolucci S, et al. The ineffective role of cathodal tDCS in enhancing the functional motor outcomes in early phase of stroke rehabilitation: an experimental trial. BioMed Res Int 2014;2014:547290. doi:10.1155/2014/547290.

[156] Lee SJ, Chun MH. Combination transcranial direct current stimulation and virtual reality therapy for upper extremity training in patients with subacute stroke. Arch Phys Med Rehabil 2014;95:431-8. doi:10.1016/j.apmr.2013.10.027.

[157] Ayerbe L, Ayis S, Crichton S, Wolfe CDA, Rudd AG. The natural history of depression up to 15 years after stroke: the South London Stroke Register. Stroke J Cereb Circ 2013;44:1105-10. doi:10.1161/STROKEAHA.111.679340.

[158] King RB. Quality of life after stroke. Stroke J Cereb Circ 1996;27:1467-72.

[159] Aström M, Asplund K, Aström T. Psychosocial function and life satisfaction after stroke. Stroke J Cereb Circ 1992;23:527-31.

[160] Silverman IE, Restrepo L, Mathews GC. Poststroke seizures. Arch Neurol 2002;59:195-201. 
[161] Opitz A, Paulus W, Will S, Antunes A, Thielscher A. Determinants of the electric field during transcranial direct current stimulation. NeuroImage 2015;109:140-50. doi:10.1016/j.neuroimage.2015.01.033.

[162] Maeda A, Yamada M, Itoh Y, Otomo E, Hayakawa M, Miyatake T. Computerassisted three-dimensional image analysis of cerebral amyloid angiopathy. Stroke J Cereb Circ 1993;24:1857-64.

[163] Vernieri F, Assenza G, Maggio P, Tibuzzi F, Zappasodi F, Altamura C, et al. Cortical neuromodulation modifies cerebral vasomotor reactivity. Stroke J Cereb Circ 2010;41:2087-90. doi:10.1161/STROKEAHA.110.583088.

[164] Nitsche MA, Paulus W. Vascular safety of brain plasticity induction via transcranial direct currents. Neurology 2015;84:556-7. doi:10.1212/WNL.0000000000001242.

[165] Parazzini M, Rossi E, Rossi L, Priori A, Ravazzani P. Numerical estimation of the current density in the heart during transcranial direct current stimulation. Brain Stimulat 2013;6:457-9. doi:10.1016/j.brs.2012.05.007.

[166] Merrill DR, Bikson M, Jefferys JGR. Electrical stimulation of excitable tissue: design of efficacious and safe protocols. J Neurosci Methods 2005;141:171-98. doi:10.1016/j.jneumeth.2004.10.020.

[167] Monte-Silva K, Kuo M-F, Hessenthaler S, Fresnoza S, Liebetanz D, Paulus W, et al. Induction of late LTP-like plasticity in the human motor cortex by repeated non-invasive brain stimulation. Brain Stimulat 2013;6:424-32. doi:10.1016/j.brs.2012.04.011.

[168] Chang W-J, Bennell KL, Hodges PW, Hinman RS, Liston MB, Schabrun SM. Combined exercise and transcranial direct current stimulation intervention for knee osteoarthritis: protocol for a pilot randomised controlled trial. BMJ Open 2015;5:e008482. doi:10.1136/bmjopen-2015-008482.

[169] Mortensen J, Figlewski K, Andersen H. Combined transcranial direct current stimulation and home-based occupational therapy for upper limb motor impairment following intracerebral hemorrhage: a double-blind randomized controlled trial. Disabil Rehabil 2015:1-7. doi:10.3109/09638288.2015.1055379.

[170] Tippett DC, Hillis AE, Tsapkini K. Treatment of Primary Progressive Aphasia. Curr Treat Options Neurol 2015;17:362. doi:10.1007/s11940-015-0362-5.

[171] Ho K-A, Bai S, Martin D, Alonzo A, Dokos S, Loo CK. Clinical Pilot Study and Computational Modeling of Bitemporal Transcranial Direct Current Stimulation, and Safety of Repeated Courses of Treatment, in Major Depression. J ECT 2015;31:226-33. doi:10.1097/YCT.0000000000000230.

[172] Cosman JD, Atreya PV, Woodman GF. Transient reduction of visual distraction following electrical stimulation of the prefrontal cortex. Cognition 2015;145:73-6. doi:10.1016/j.cognition.2015.08.010.

[173] Fitz NS, Reiner PB. The challenge of crafting policy for do-it-yourself brain stimulation. J Med Ethics 2015;41:410-2. doi:10.1136/medethics-2013-101458.

[174] de Berker AO, Bikson M, Bestmann S. Predicting the behavioral impact of transcranial direct current stimulation: issues and limitations. Front Hum Neurosci 2013;7:613. doi:10.3389/fnhum.2013.00613. 
[175] Jacobson L, Koslowsky M, Lavidor M. tDCS polarity effects in motor and cognitive domains: a meta-analytical review. Exp Brain Res 2012;216:1-10. doi:10.1007/s00221-011-2891-9.

[176] Brunoni AR, Ferrucci R, Bortolomasi M, Scelzo E, Boggio PS, Fregni F, et al. Interactions between transcranial direct current stimulation (tDCS) and pharmacological interventions in the Major Depressive Episode: findings from a naturalistic study. Eur Psychiatry J Assoc Eur Psychiatr 2013;28:356-61. doi:10.1016/j.eurpsy.2012.09.001.

[177] Hagenacker T, Bude V, Naegel S, Holle D, Katsarava Z, Diener H-C, et al. Patient-conducted anodal transcranial direct current stimulation of the motor cortex alleviates pain in trigeminal neuralgia. J Headache Pain 2014;15:78. doi:10.1186/1129-2377-15-78.

[178] Charvet LE, Kasschau M, Datta A, Knotkova H, Stevens MC, Alonzo A, et al. Remotely-supervised transcranial direct current stimulation (tDCS) for clinical trials: guidelines for technology and protocols. Front Syst Neurosci 2015;9:26. doi:10.3389/fnsys.2015.00026.

[179] Kasschau M, Sherman K, Haider L, Frontario A, Shaw M, Datta A, et al. A Protocol for the Use of Remotely-Supervised Transcranial Direct Current Stimulation (tDCS) in Multiple Sclerosis (MS). J Vis Exp 2015. doi:10.3791/53542.

[180] Steenbergen L, Sellaro R, Hommel B, Lindenberger U, Kühn S, Colzato LS. "Unfocus" on foc.us: commercial tDCS headset impairs working memory. Exp Brain Res 2015. doi:10.1007/s00221-015-4391-9.

[181] Wexler A. The practices of do-it-yourself brain stimulation: implications for ethical considerations and regulatory proposals. J Med Ethics 2015. doi:10.1136/medethics-2015-102704.

[182] Calabrese EJ. Preconditioning is hormesis part I: Documentation, dose-response features and mechanistic foundations. Pharmacol Res 2016. doi:10.1016/j.phrs.2015.12.021.

[183] Calabrese EJ, Dhawan G, Kapoor R, Iavicoli I, Calabrese V. HORMESIS: A Fundamental Concept with Widespread Biological and Biomedical Applications. Gerontology 2015. doi:10.1159/000441520.

[184] Baba T, Kameda M, Yasuhara T, Morimoto T, Kondo A, Shingo T, et al. Electrical stimulation of the cerebral cortex exerts antiapoptotic, angiogenic, and antiinflammatory effects in ischemic stroke rats through phosphoinositide 3kinase/Akt signaling pathway. Stroke J Cereb Circ 2009;40:e598-605. doi:10.1161/STROKEAHA.109.563627.

[185] Peruzzotti-Jametti L, Cambiaghi M, Bacigaluppi M, Gallizioli M, Gaude E, Mari $\mathrm{S}$, et al. Safety and efficacy of transcranial direct current stimulation in acute experimental ischemic stroke. Stroke J Cereb Circ 2013;44:3166-74. doi:10.1161/STROKEAHA.113.001687.

[186] Sattler V, Acket B, Raposo N, Albucher J-F, Thalamas C, Loubinoux I, et al. Anodal tDCS Combined With Radial Nerve Stimulation Promotes Hand Motor Recovery in the Acute Phase After Ischemic Stroke. Neurorehabil Neural Repair 2015;29:743-54. doi:10.1177/1545968314565465. 
[187] Tahtis V, Kaski D, Seemungal BM. The effect of single session bi-cephalic transcranial direct current stimulation on gait performance in sub-acute stroke: A pilot study. Restor Neurol Neurosci 2014;32:527-32. doi:10.3233/RNN-140393.

[188] Fusco A, Iosa M, Venturiero V, De Angelis D, Morone G, Maglione L, et al. After vs. priming effects of anodal transcranial direct current stimulation on upper extremity motor recovery in patients with subacute stroke. Restor Neurol Neurosci 2014;32:301-12. doi:10.3233/RNN-130349. 

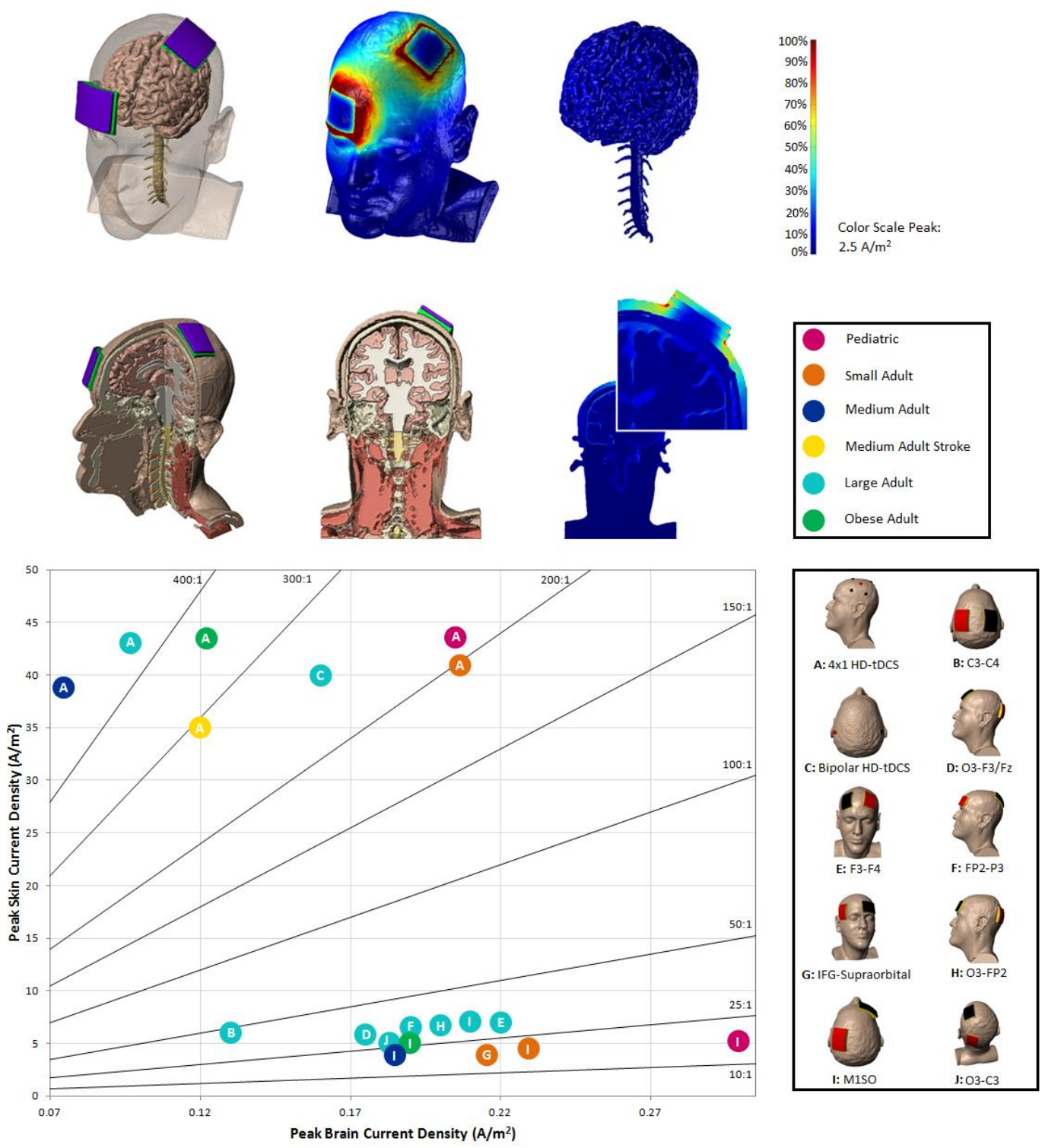

Figure 1: Computational models predict skin current density to brain current density, and so their ratio. An exploratory analysis compared a range of montages on extremes of head anatomy (e.g. pediatric to obese, healthy and stroke). Additional models were solved to increase the depth of the study (methods of computational forward modeling are described in detail in Figure 3). Models included some or all of the following tissue masks: skin (0.465 $\mathrm{S} / \mathrm{m})$, fat $(0.025 \mathrm{~S} / \mathrm{m})$, bone $(0.01 \mathrm{~S} / \mathrm{m})$, CSF $(1.65 \mathrm{~S} / \mathrm{m})$, gray matter $(0.276 \mathrm{~S} / \mathrm{m})$, white matter $(0.126 \mathrm{~S} / \mathrm{m})$, intervertebral discs $(0.16133 \mathrm{~S} / \mathrm{m})$, ligament $(0.250922 \mathrm{~S} / \mathrm{m})$, spinal cord $(0.171267 \mathrm{~S} / \mathrm{m})$, air $\left(1 \times 10^{-15} \mathrm{~S} / \mathrm{m}\right)$, electrode $\left(5.99 \times 10^{7} \mathrm{~S} / \mathrm{m}\right)$, sponge $(1.4 \mathrm{~S} / \mathrm{m})$, and gel $(4.0 \mathrm{~S} / \mathrm{m})$. The review involved nineteen combinations of six different head types (pediatric, small adult, medium adult, medium adult stroke, large adult, and obese adult) and ten different electrode montages (two using HD electrodes and eight using $5 \times 7 \mathrm{~cm}$ sponge pad electrodes). (Top) Because electrodes are placed on the scalp during tDCS, and due to the conductivity and anatomy of the underlying tissue, a majority of the current does not reach the brain and the fraction that does reach the brain is diffused. The current density in skin is thus invariably higher than in the brain. (Bottom) The maximum current density in the skin and the brain (and their ratio) depends on several factors including the electrode montage. For a single head, the ratio is predicted for various conventional and HD montages. The skin to brain ratio varies from greater than 10:1 to 400:1.

The maximum brain current density was $0.23 \mathrm{~A} / \mathrm{m} 2$ for a small adult head and $0.32 \mathrm{~A} / \mathrm{m} 2$ for pediatric head (Image adapted from Tyler et al, in preparation) 


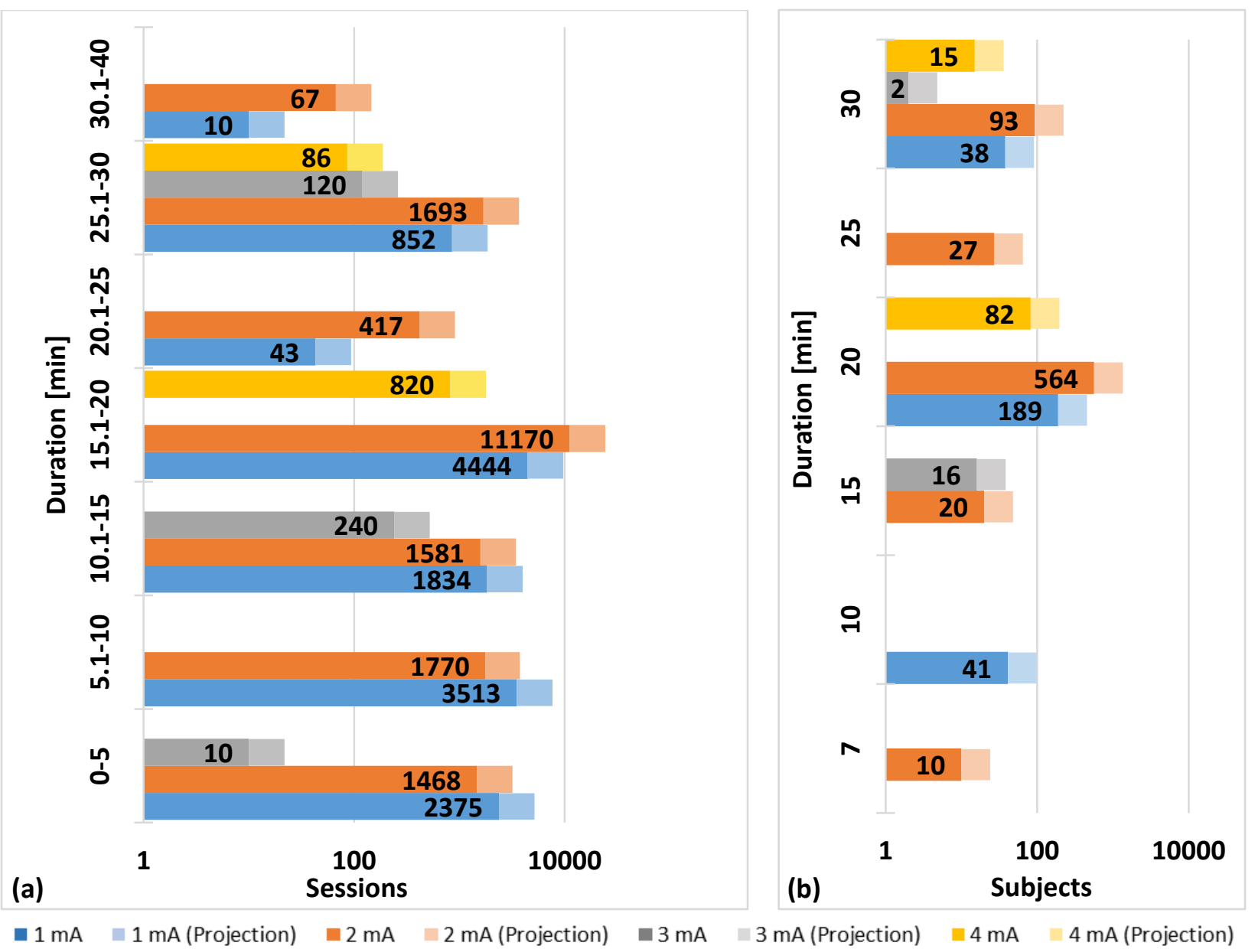

Figure 2: Number of Sessions by Duration (a) and Number of Subjects in Repeated Sessions by Duration (b) Interim analysis of total sessions and dose in tDCS literature of published trials meeting our inclusion criterion. We searched the Pubmed database with the key words "transcranial direct current stimulation" limiting to papers published in English. We only included studies that met the following inclusion criteria: (1) used tDCS, (2) tested on human subjects, (3) reported original research, (4) used an electrolyte-soaked absorbent material, (5) clearly reported dosage information, and (6) published before July 2013. 488 of 1072 papers were considered. Of those, tDCS dosage and number of sessions were extracted. tDCS dosage includes current intensity, electrode size, duration, and position (not reported here). Number of sessions refers to the number of tDCS procedures completed (e.g. number of subjects times sessions per subject). If one subject underwent more than 4 sessions in one week, it was further classified as a repeated session. Data from $\sim 55 \%$ of all original tDCS publications extracted using a combination of automated lexica analysis and manual screening (solid bar) and extrapolated based on update tDCS publication volume (lighter bar) - therefore the solid bars represent verified statistics until July 2013 while lighter bar represents prediction given fixed distribution of sessions in all papers to-date. We extracted the following dose parameters: Current applied, duration of session, current density at electrodes, and for studies with repeated sessions (three to seven treatments per week for at least one week) the number of sessions. If a clinical trial tested more than one tDCS condition per subject (e.g. anode vs. cathode over M1 separated by one week) these were considered separate sessions since the study design intended no carry-over effects and since the results of each session provide evidence for safety. From each qualified trial the total number of sessions and the parameters (intensity, duration, current density) for each session where determined. Therefore the total number of sessions applied at any given parameter (e.g. current of $2 \mathrm{~mA}$ ) or combination of parameters (e.g. current of $2 \mathrm{~mA}$ and duration of 20 minutes) for tDCS trials is known. For repeated sessions, we aggregated data by number of subjects. Cumulative data is plotted under the assumption that increasing intensity or duration does not decrease risk, such that sessions at $2 \mathrm{~mA}$ support safety at $1 \mathrm{~mA}$. This assumption presumes a monotonic dose-safety response curve and does not apply to efficacy. 
A

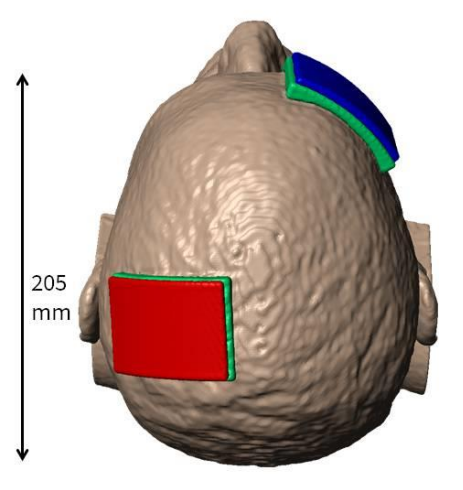

C

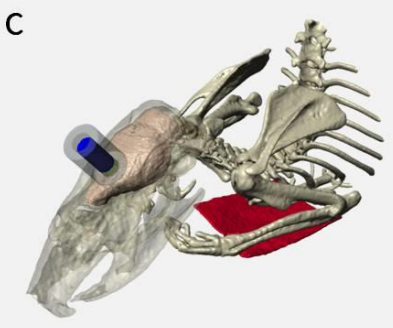

Fritsch et al. $4 \mathrm{~mm} \mathrm{D}$ $600 \mu \mathrm{A}, 20 \mathrm{~min}$

E

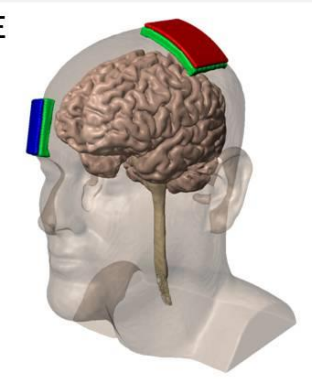

M1-SO

$5 \times 7 \mathrm{~cm}$

$2 \mathrm{~mA}, 20 \mathrm{~min}$

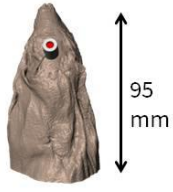

D
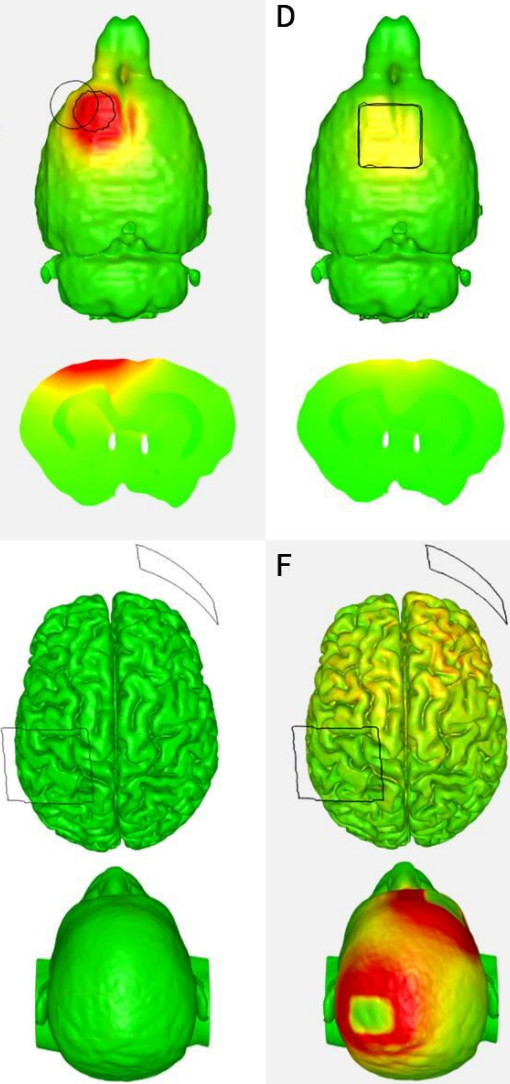

$\mathrm{F}$

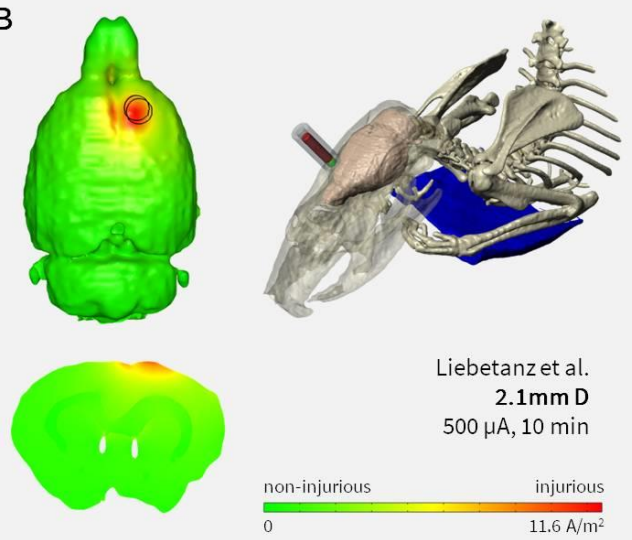

B

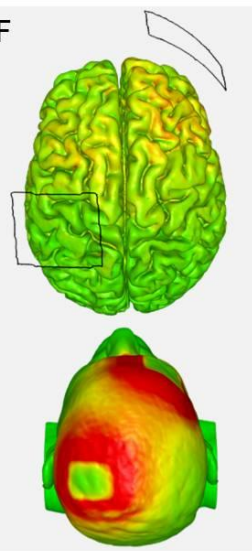

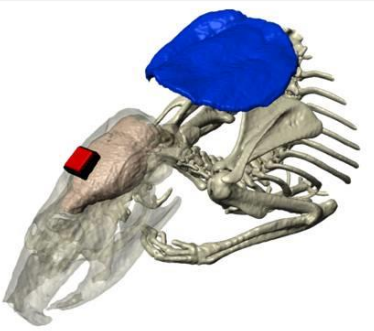

Jankord et al.

$5 \times 5 \mathrm{~mm}$

$500 \mu \mathrm{A}, 60 \mathrm{~min}$

Figure 3: Finite Element models comparing a common tDCS montage in human and three DCS montages in animal model. Animal data indicates possible injury at electric field thresholds over an order of magnitude above those generated by conventional tDCS protocols. (A) The evident difference in gross anatomy between human and rat is considered in computational models based on high-resolution MRI. (B, C, D) Lesion threshold in rat brain as reported by three different groups using modestly varied methods. The predicted minimum induced current density for brain lesions ranged from $12,17,6.3 \mathrm{~A} / \mathrm{m}^{2}$ (corresponding to electric fields of 42,61 , to $23 \mathrm{~V} / \mathrm{m}$ ) for the montages used by Liebetanz et al. (B) Fritsch et al (C) and Jankord et al. (D) respectively. In contrast to human tDCS, epicranial stimulation of the rat brain (stimulation applied over skull) resulted in higher cortical electric fields for the same input current, magnified by the smaller head anatomy. (E) Typical human tDCS driven by $2 \mathrm{~mA}$ at the electrodes resulted in $0.096 \mathrm{~A} / \mathrm{m}^{2}(0.35 \mathrm{~V} / \mathrm{m})$ on the cortex. (F) To match the cortical lesion threshold found in Liebetanz et al., $120 \mathrm{~mA}$ would have to be applied in human. We note however that current density in the skin would be much higher than in the brain, such that skin injury would potentially manifest well before the risk of brain injury. (Simulations and data adopted from Liebetanz et al 2009, Frisch unpublished observations, Jankord unpublished observations, Truong unpublished observations). 


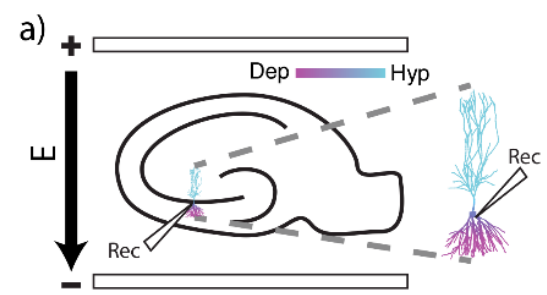

b)

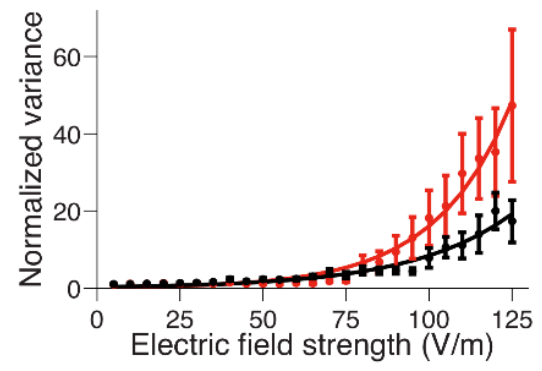

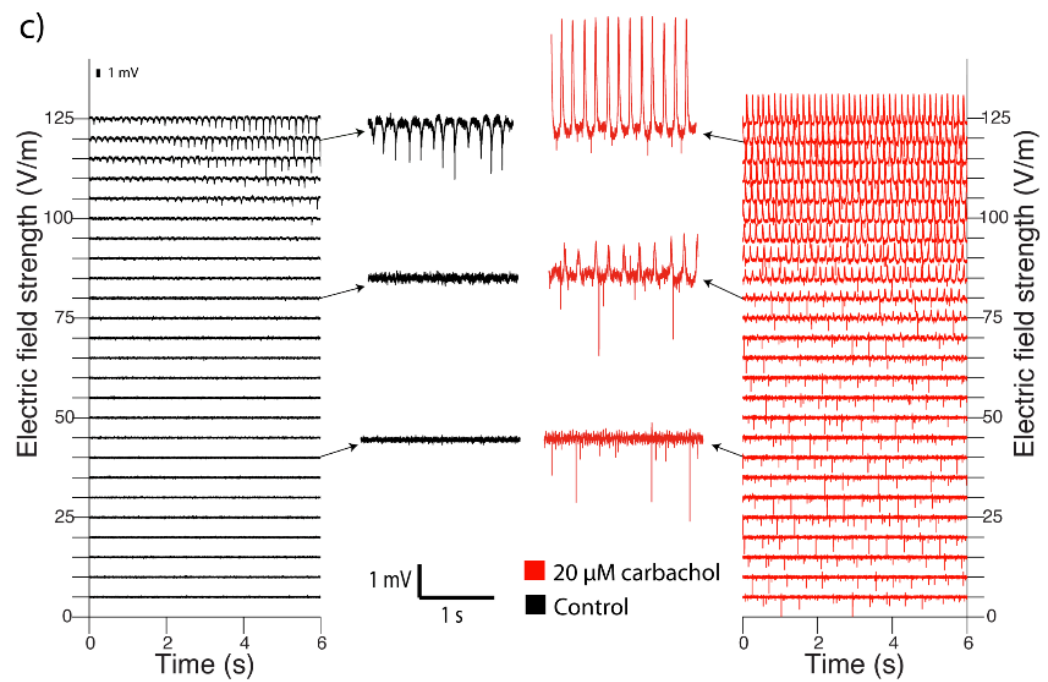

Figure 4: Endogenous oscillation activity does not significantly reduce epileptic threshold compared to quiescent state in brain slices. The electric field intensity needed to generate epileptiform activity can be systematically investigated in bran slices. Uniform electric field up to $125 \mathrm{~V} / \mathrm{m}$ were tested, but note tDCS produces electrics fields $<1 \mathrm{~V} / \mathrm{m}$. a) Rat hippocampal slices were prepared according to Reato et al. 2010 and stimulated with electric fields (E) of varying intensity (10 s duration) along the somatodendritic axis of CA3 pyramidal neurons. 20 $\mu \mathrm{M}$ carbachol was added to the aCSF superfusate to induce spontaneous neural activity. Slices were either quiescent (normal aCSF superfusate; Black traces) or oscillating (+carbochal ACSF, Red traces). Inset is a computational model showing the membrane polarization profile of a pyramidal neuron in an electric field (Dep, depolarized; Hyp, hyperpolarized). b) Epileptiform activity was quantified as the average variance of the voltage signal during stimulation, normalized to the average variance during the 10 seconds before stimulation. Mean +/- SEM across slices is plotted for each electric field intensity. Epileptic threshold for each slice was quantified as the electric field intensity required to produce a normalized variance $>10$. There was no significant difference in epileptic threshold between carbcahol and control conditions $(\mathrm{p}=0.48$; carbachol $98.3+/-15.61 \mathrm{~V} / \mathrm{m} \mathrm{n}=10$; control $104+/-14.97$ $\mathrm{V} / \mathrm{m} \mathrm{n}=9$ ). c) Sample traces during stimulation at each electric field intensity (Left column black-traces normal aCSF Right column red-traces +Carbachol). Highlighted traces at 40,80, and $120 \mathrm{~V} / \mathrm{m} /$ Even at $40 \mathrm{~V} / \mathrm{m}(>40 \mathrm{x}$ electric fields generated by tDCS) no epileptiform activity compared to baseline (quiescent or oscillation) was detected (Kronberg at al. unpublished data). 


\section{A Limited data points from studies using varied methods}

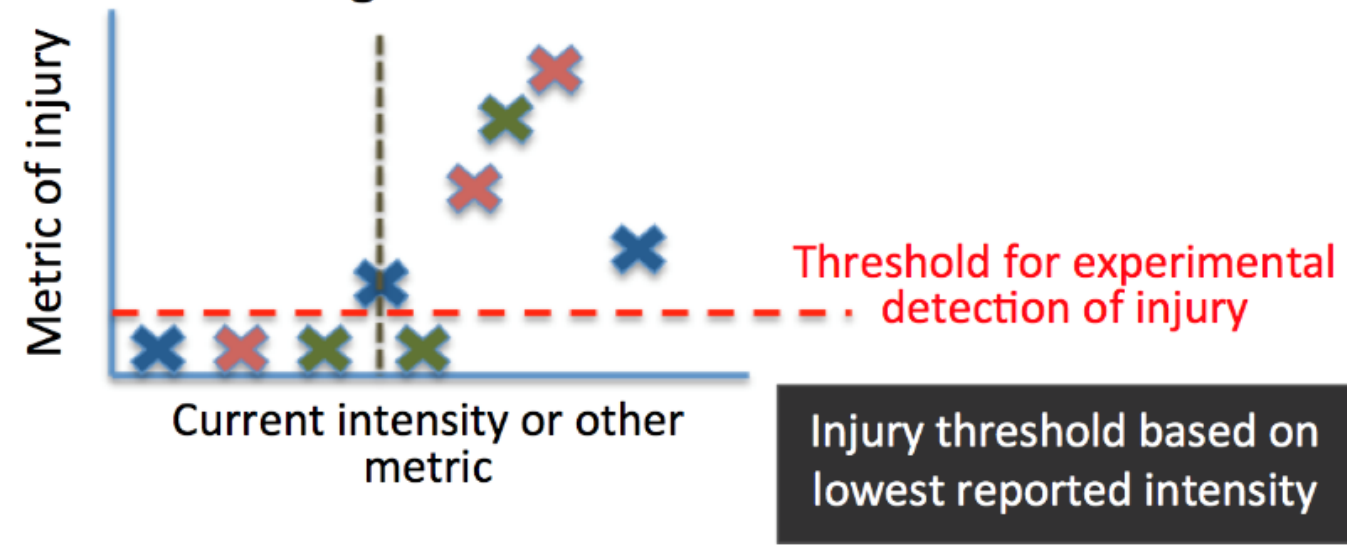

B Dose response curve assumptions

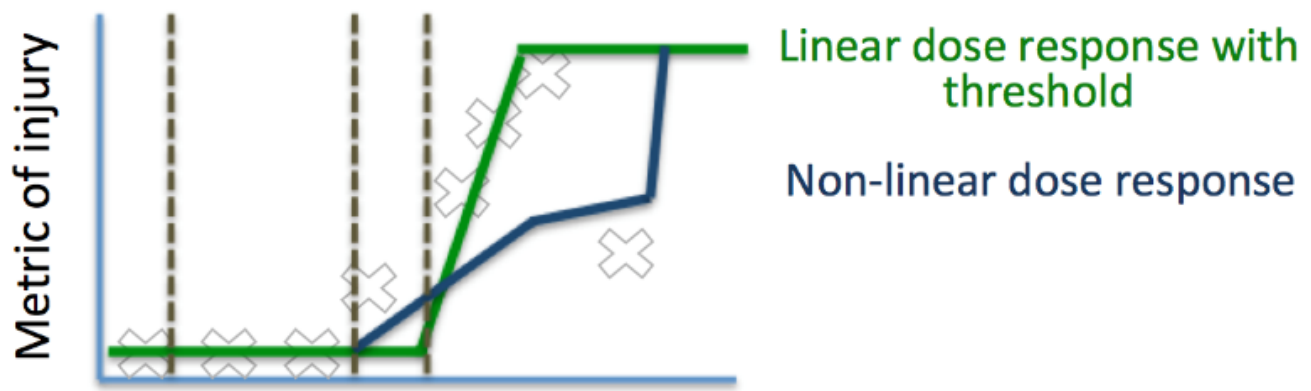

\section{Current intensity or other} metric

\section{Injury threshold based on varied dose response curves}

Figure 5: Dose response curves and assumptions. While all existing data from animal models of epicranial DCS suggest injury thresholds are well above conventional tDCS protocols, data across intensities is limited and various models (e.g. animal species and strain) and metrics (e.g. histological stains) of injury are used (represented schematically by color of X). (A) The approach taken in this review was limited to empirical data based on the lowest reported injury threshold as a current intensity. This approach is limited by the sensitivity of the experimental measure for injury and other details of the experiment and limitations of animal models. But, none-the-less, the single lowest reported injury intensity represents at this time a condition clinical tDCS should not approach. (B) Alternatively, various dose response curves can be fit to one or more data sets. This approach is especially sensitive to assumptions about threshold, even when existing data from a given trial appears to fit a specific curve reliably. Using any extrapolated function will inevitably lead to a projected injury threshold below the lowest experimentally measured threshold. In summary, in the absence of an established mechanism for injury and/or a justified doseresponse curve, it is difficult to reliably extrapolate below existing experimental determined thresholds for injury in animals - which are substantially above intensities generated by conventional tDCS. 


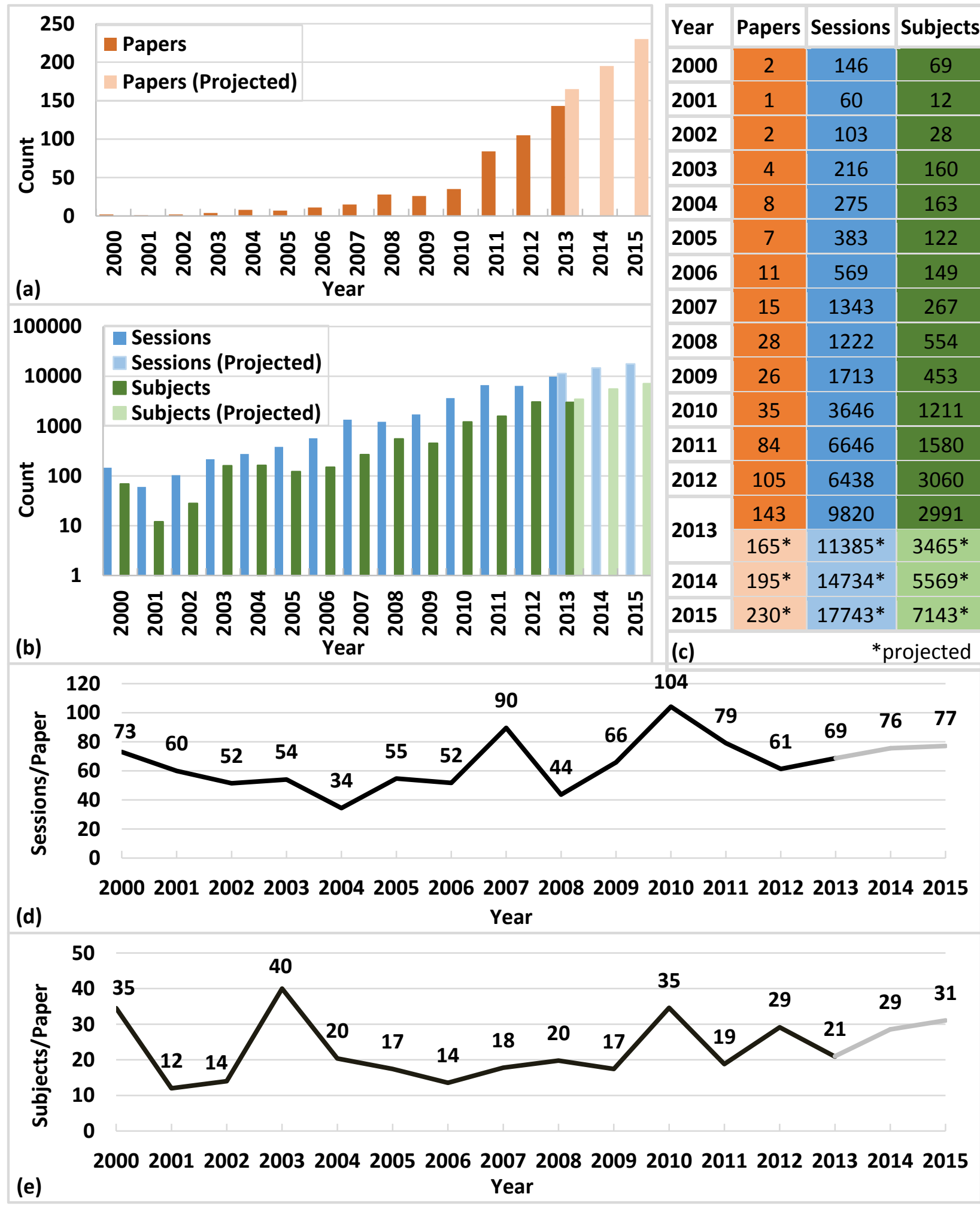

Figure 6: Visual display of original Research papers published (a), along with Sessions performed and Subjects tested by annum (b). Quantitative data for both are listed separately (c). The ratio of sessions/paper per year is also given (d) and subjects/paper per year (e). Both ratios were calculated using the average amount of sessions or subjects per year. Lighter colors indicate projections (see rationale in Figure 2). 
(a)

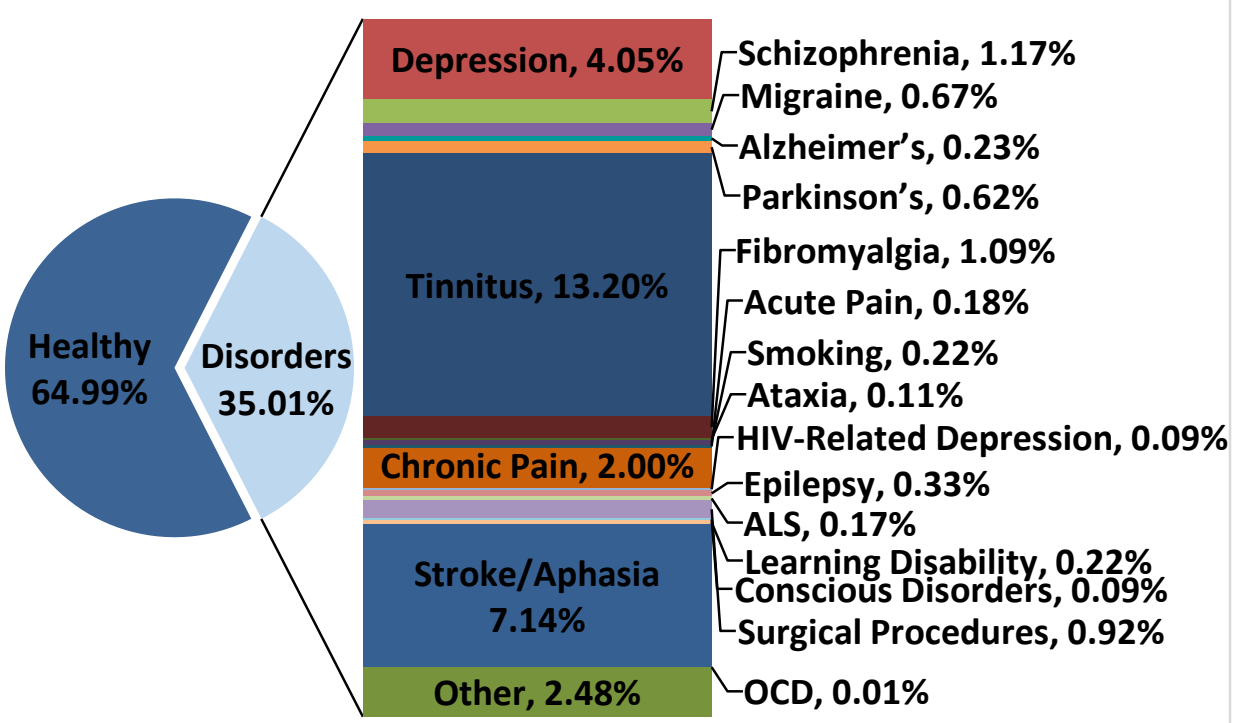

(b)
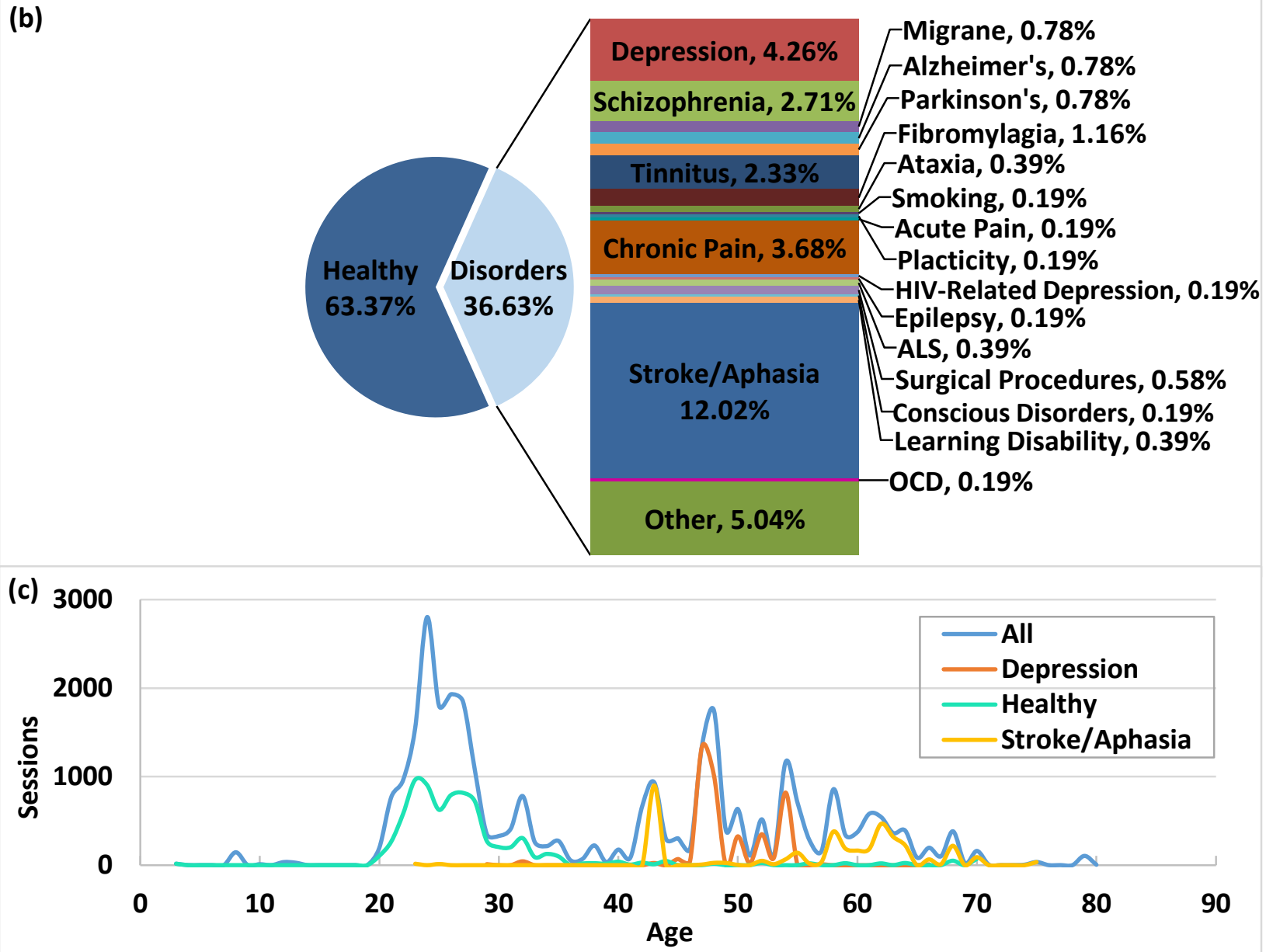

Figure 7: tDCS subject Demographic Charts. Medical conditions of subjects treated with tDCS, as reported in the papers analyzed (a). Papers published by medical conditions of subjects (b). Number of sessions given by average age (c) for all sessions that reported age, as well as sessions with subjects with depression, stroke/aphasia, only healthy subjects. Age was calculated by taking the average age of a subject group or using the age range to calculate an approximate average age. 

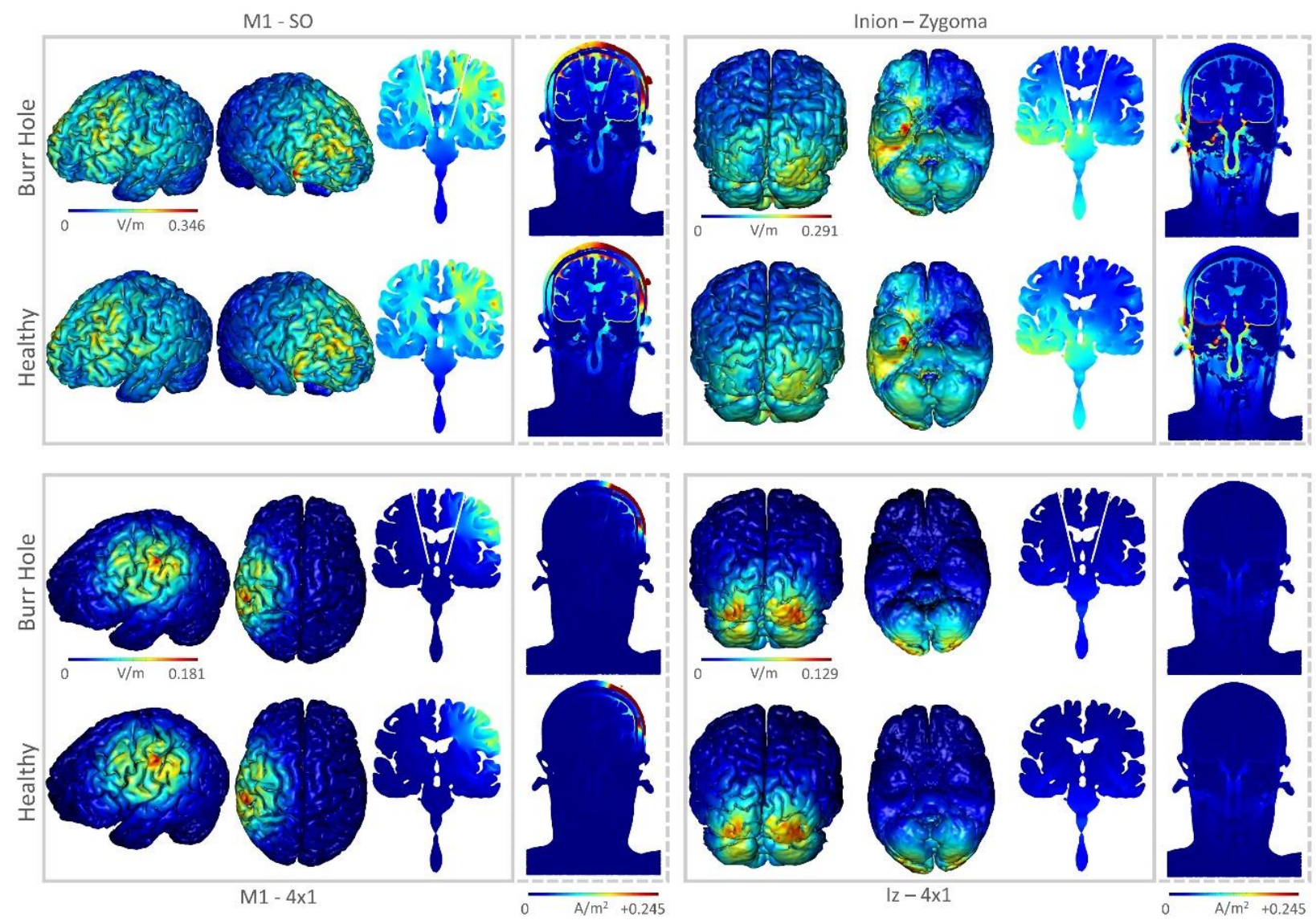

Figure 8: Computer simulation of brain current flow produced by tDCS in the presence of an idealized Deep Brain Stimulation lead. Finite Element models of tDCS with and without burrhole defects typical in subthalamic nucleus deep brain stimulation. Common sponge (conventional) and HD-tDCS montages for motor and cerebellar stimulation are compared. Four montages are simulation M1-SO (top left) and Inion-Zygoma (top right), 4x1 HDtDCS over M1 (bottom left) and 4x1 HD-tDCS over Iz (bottom right). In each care current flow produced through the head in the "healthy" case (intact skull and brain, no implant) is compared to a worst-case scenario where two "burr holes" through the skin, skull and brain are created and fully filled with CSF. Fluid filled burr holes draw a greater amount of current density than what would normally exist with healthy tissue (dashed images). However, peak current density and electric field are minimally affected (less than two fold). HD configurations have lower deep brain electric field intensities in general in addition to being more confined. Thus, even under these worst-case burr-hole conditions, there are only moderate changes in overall brain current flow patterns, and no large change in peak electric field. This suggest that under more realistic conditions (e.g. with the implant in place instead of CSF, skin present and the skull largely sealed) the presence of a DBS lead would not significantly change resulting brain current flow. Evidently, all models are limited to the assumption made and this simulation (Truong, Bikson et al., in preparation). 